\title{
SN 2005bf: A POSSIBLE TRANSITION EVENT BETWEEN TYPE Ib/c SUPERNOVAE AND GAMMA-RAY BURSTS
}

\author{
Gastón Folatelli, ${ }^{1}$ Carlos Contreras, ${ }^{1}$ M. M. Phillips, ${ }^{1}$ S. E. Woosley, ${ }^{2}$ Sergei Blinnikov, ${ }^{3}$ Nidia Morrell, ${ }^{1,4}$ \\ Nicholas B. Suntzeff, ${ }^{5}$ Brian L. Lee, ${ }^{6}$ Mario Hamuy, ${ }^{1,7}$ Sergio González, ${ }^{1}$ Wojtek Krzeminski, ${ }^{1}$ Miguel Roth, ${ }^{1}$ \\ Weidong Li, ${ }^{8}$ Alexei V. Filippenko, ${ }^{8}$ Ryan J. Foley, ${ }^{8}$ W. L. Freedman, ${ }^{9}$ Barry F. Madore, ${ }^{9,10}$ \\ S. E. Persson, ${ }^{9}$ David Murphy,${ }^{9}$ Samuel Boissier, ${ }^{9}$ Gaspar Galaz, ${ }^{11}$ Luis González, ${ }^{7}$ \\ P. J. McCarthy, ${ }^{9}$ Andrew McWilliam, ${ }^{9}$ and Wojtek PyCh ${ }^{12}$ \\ Received 2005 September 16; accepted 2005 December 15
}

\begin{abstract}
We present $u^{\prime} g^{\prime} r^{\prime} i^{\prime} B V$ photometry and optical spectroscopy of the Type $\mathrm{Ib} / \mathrm{Ic} \mathrm{SN} 2005 \mathrm{bf}$ covering the first $\sim 100$ days following discovery. The $u^{\prime} g^{\prime} B V$ light curves displayed an unprecedented morphology among Type $\mathrm{Ib} / \mathrm{Ic}$ supernovae, with an initial maximum some 2 weeks after discovery and a second, main maximum about 25 days after that. The bolometric light curve indicates that SN 2005 bf was a remarkably luminous event, radiating at least $6.3 \times 10^{42} \mathrm{ergs} \mathrm{s}^{-1}$ at maximum light and a total of $2.1 \times 10^{49}$ ergs during the first 75 days after the explosion. Spectroscopically, SN 2005 bf underwent a unique transformation from a Type Ic-like event at early times to a typical Type Ib supernova at later phases. The initial maximum in $u^{\prime} g^{\prime} B V$ was accompanied by the presence in the spectrum of high-velocity ( $\left.>14,000 \mathrm{~km} \mathrm{~s}^{-1}\right)$ absorption lines of $\mathrm{Fe}$ п, $\mathrm{Ca}$ II, and $\mathrm{H}$ I. The photospheric velocity derived from spectra at early epochs was below $10,000 \mathrm{~km} \mathrm{~s}^{-1}$, which is unusually low compared with ordinary Type Ib supernovae. We describe one-dimensional computer simulations that attempt to account for these remarkable properties. The most favored model is that of a very energetic $\left(2 \times 10^{51} \mathrm{ergs}\right)$, asymmetric explosion of a massive $\left(8.3 M_{\odot}\right)$ Wolf-Rayet WN star that had lost most of its hydrogen envelope. We speculate that an unobserved relativistic jet was launched producing a two-component explosion consisting of (1) a polar explosion containing a small fraction of the total mass and moving at high velocity and (2) the explosion of the rest of the star. At first, only the polar explosion is observed, producing the initial maximum and the high-velocity absorption-line spectrum resembling a Type Ic event. At late times, this fast-moving component becomes optically thin, revealing the more slowly moving explosion of the rest of the star and transforming the observed spectrum to that of a typical Type $\mathrm{Ib}$ supernova. If this scenario is correct, then SN 2005 bf is the best example to date of a transition object between normal Type $\mathrm{Ib} / \mathrm{Ic}$ supernovae and $\gamma$-ray bursts.
\end{abstract}

Subject headings: gamma rays: bursts — supernovae: individual (SN 2005bf)

\section{INTRODUCTION}

After years of relative neglect, Type Ib/Ic supernovae (SNe; see Filippenko 1997 for a discussion of SN spectral types) are today the object of considerable observational and theoretical attention. This boom is largely due to the discovery that certain $\gamma$-ray bursts (GRBs) are associated with highly energetic Type Ic events commonly referred to as "hypernovae" (e.g., see Stanek

\footnotetext{
1 Las Campanas Observatory, Carnegie Observatories, Casilla 601, La Serena, Chile.

2 Department of Astronomy, University of California, Santa Cruz, CA 95064.

3 Max Planck Institute for Astrophysics, 85741 Garching, Germany; and Alikhanov Institute for Theoretical and Experimental Physics, 117218 Moscow, Russia.

${ }^{4}$ Member of the Carrera del Investigador Científico, CONICET, Argentina; on leave from Facultad de Ciencias Astronómicas y Geofísicas, Universidad Nacional de La Plata.

5 Cerro Tololo Inter-American Observatory, Casilla 603, La Serena, Chile.

${ }^{6}$ Department of Astronomy and Astrophysics, University of Toronto, 60 St. George Street, Toronto, ON M5S 3H8, Canada.

${ }^{7}$ Universidad de Chile, Departamento de Astronomía, Casilla 36-D, Santiago, Chile.

${ }^{8}$ Department of Astronomy, University of California, Berkeley, CA 94720-3411.

9 Observatories of the Carnegie Institution of Washington, 813 Santa Barbara Street, Pasadena, CA 91101

${ }^{10}$ Infrared Processing and Analysis Center, Jet Propulsion Laboratory, California Institute of Technology, Pasadena, CA 91125.

11 Departamento de Astronomía y Astrofísica, Pontificia Universidad Católica de Chile, Casilla 306, Santiago 22, Chile.

12 Copernicus Astronomical Center, Bartycka 18, PL-00716 Warsaw, Poland.
}

et al. 2005 and references therein). Note, however, that this term has been expanded by some authors to include essentially any Type Ic SN with inferred high luminosity or unusually broad lines, whether or not it was associated with a GRB.

It is universally agreed that Type $\mathrm{Ib} / \mathrm{Ic} \mathrm{SNe}$, as well as the transitional Type IIb SNe, are produced by the core collapse of a massive star that has lost most or all of its hydrogen envelope before exploding (e.g., see Filippenko 2005 and references therein). Taken together as a class, the light curves of Type $\mathrm{Ib} / \mathrm{Ic} / \mathrm{IIb} \mathrm{SNe}$ display a significant range in luminosities and morphologies, although there are subclasses of events that are relatively homogeneous in their properties (Clocchiatti \& Wheeler 1997). Unfortunately, only a handful of Type Ib/Ic SNe have been observed significantly before maximum (e.g., Foley et al. 2003); thus, little is known of their photometric and spectroscopic properties at the earliest epochs following outburst.

At the Las Campanas Observatory ( $\mathrm{LCO}$ ), we have embarked on a multiyear observational program - the Carnegie Supernova Program (CSP) - to obtain follow-up photometry and spectroscopy of SNe of all types (see Hamuy et al. 2006, hereafter Paper I). One of the many goals of this project is to obtain uniform, highprecision observations of nearby Type $\mathrm{Ib} / \mathrm{Ic} / \mathrm{Ilb} \mathrm{SNe}$ in order to improve our knowledge of the optical and near-infrared characteristics of these explosions. Highest priority is given to $\mathrm{SNe}$ discovered soon after outburst, since it is at these early epochs when the largest range in photometric and spectroscopic properties is likely to be observed. 


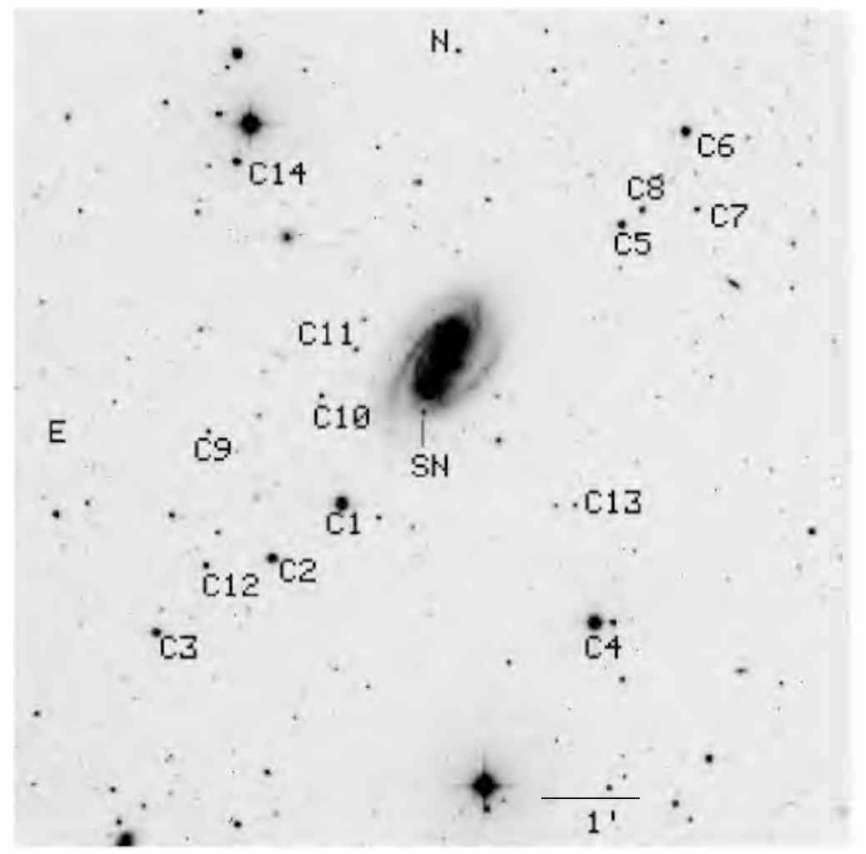

Flg. 1.-Field of SN $2005 \mathrm{bf}$ observed with the Swope $1 \mathrm{~m}$ telescope at LCO and a $V$ filter. North is up and east is to the left. The supernova is marked to the southeast of the host-galaxy nucleus. Fourteen comparison stars used to derive differential photometry of the SN are labeled. The image scale is shown near the bottom.

One of the Type $\mathrm{Ib} / \mathrm{Ic}$ events to be studied in detail as part of the CSP was SN 2005bf, which was independently discovered (Monard et al. 2005) on 2005 April 6 (UT dates are used throughout this paper) by L. A. G. Monard at the Bronberg Observatory in South Africa and by M. Moore and W. Li during the Lick Observatory Supernova Search (LOSS; Li et al. 2000; Filippenko et al. 2001; Filippenko 2005). The SN was located 11 " 7 east and 32" 6 south of the nucleus of MCG $+00-27-5$, an SB galaxy with a heliocentric recession velocity of $5670 \mathrm{~km} \mathrm{~s}^{-1}$ (Falco et al. 1999). According to Monard, nothing was visible at the position of the SN on an unfiltered image taken on March 12 with a limiting magnitude of 18.5. Moore and Li report no detection to a limiting unfiltered magnitude of 19.5 on an image from March 15 and a marginal detection at 18.8 on March 30.
It was likely, therefore, that the explosion occurred between March 15 and 29.

Initial CSP spectroscopy revealed that SN 2005bf was a Type Ic event (Morrell et al. 2005), and optical photometry confirmed that the SN had, indeed, been caught on the rising part of the light curve. An intensive program of follow-up photometric and spectroscopic observations was initiated, which unexpectedly revealed an unusual preliminary rise and fall in the $u^{\prime}, g^{\prime}, B$, and $V$ light curves preceding by $\sim 25$ days the main maxima in these bands (Hamuy et al. 2005). Spectra obtained just before the time of the main maximum also revealed that SN 2005bf had transformed into a Type Ib or transitional Type IIb event with strong He I lines (Wang \& Baade 2005).

In this paper, we present the CSP observations of SN 2005bf and discuss these in the context of possible models of the star that produced this peculiar and, thus far, unique explosion.

\section{PHOTOMETRY}

Most of our optical photometry was obtained with the Swope $1 \mathrm{~m}$ telescope at LCO, using a SITe CCD and a set of Sloan Digital Sky Survey (SDSS) $u^{\prime} g^{\prime} r^{\prime} i^{\prime}$ and Johnson $B V$ filters (Fukugita et al. 1996; Bessell 1990). We read a section of $1200 \times 1200$ pixels from the $C C D$, which, at a scale of $0^{\prime \prime} 435$ pixel $^{-1}$. yielded a field of view of $8.7 \times 8 !^{\prime} 7$. Typical image quality ranged between $1^{\prime \prime}$ and $2^{\prime \prime}$ (FWHM). A photometric sequence of comparison stars in the SN field was calibrated with the Swope telescope from observations of standard stars of Landolt (1992) and Smith et al. (2002) during four photometric nights. Figure 1 shows the SN field and the selected comparison stars. Table 1 lists the average $u^{\prime} g^{\prime} r^{\prime} i^{\prime} B V$ magnitudes derived for these stars. SN magnitudes in the standard SDSS+Johnson system were obtained differentially relative to the comparison stars using point-spread function (PSF) photometry. On every image, a PSF was fitted to the SN and comparison stars within a radius of $3^{\prime \prime}$. We refer to Paper I for further details about the instrument and measurements.

Five unfiltered LOSS images obtained with the $0.8 \mathrm{~m}$ Katzman Automatic Imaging Telescope (KAIT; Filippenko et al. 2001; Filippenko 2005) were included in our analysis because they allow us to study the very early stages of the SN. After some experimentation we found that the unfiltered instrumental magnitudes of the local standards could be satisfactorily transformed with a simple additive zero point to the $r^{\prime}$ standard system with a dispersion $\lesssim 0.05 \mathrm{mag}$. The comparison of the resulting LOSS magnitudes of

TABLE 1

Photometry of the Comparison Stars in the Field of SN 2005bf

\begin{tabular}{|c|c|c|c|c|c|c|}
\hline Star ID & $u^{\prime}$ & $g^{\prime}$ & $r^{\prime}$ & $\dot{i}^{\prime}$ & $B$ & $V$ \\
\hline $\mathrm{C} 1 .$. & ... & $\ldots$ & $\ldots$ & $\therefore$ & $14.657(016)$ & $\therefore$ \\
\hline . & $16.360(021)$ & $15.186(015)$ & $14.762(015)$ & $14.630(015)$ & $15.545(009)$ & $14.943(010)$ \\
\hline C3.. & $19.203(016)$ & $16.529(008)$ & $15.463(009)$ & $15.067(009)$ & $17.141(009)$ & $15.963(008)$ \\
\hline $\mathrm{C} 4 \ldots$ & $17.105(016)$ & $\ldots$ & $\ldots$ & $\ldots$ & $15.081(016)$ & $\ldots$ \\
\hline C5 $5 \ldots \ldots$ & $19.988(034)$ & $17.292(009)$ & $16.115(008)$ & $15.624(008)$ & $17.938(010)$ & $16.675(007)$ \\
\hline 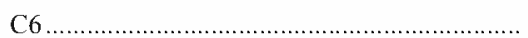 & $16.254(020)$ & $15.147(011)$ & $14.753(011)$ & $14.603(011)$ & $15.476(009)$ & $14.905(010)$ \\
\hline . & $21.254(205)$ & $18.924(009)$ & $18.013(008)$ & $17.676(008)$ & $19.446(016)$ & $18.434(008)$ \\
\hline C8 .................. & $18.328(013)$ & $17.040(008)$ & $16.529(008)$ & $16.328(008)$ & $17.433(009)$ & $16.743(007)$ \\
\hline C9........ & $22.883(797)$ & $20.755(028)$ & $19.362(018)$ & $18.302(009)$ & $19.504(477)$ & $19.957(024)$ \\
\hline $\mathrm{C} 10 \ldots \ldots$ & $19.416(033)$ & $18.473(009)$ & $18.125(008)$ & $18.022(013)$ & $18.770(011)$ & $18.259(008)$ \\
\hline $\mathrm{C} 11$ & $20.418(046)$ & $18.628(008)$ & $17.953(008)$ & $17.691(008)$ & $19.104(015)$ & $18.247(008)$ \\
\hline C $12 .$. & $19.604(044)$ & $17.845(008)$ & $17.176(008)$ & $16.939(008)$ & $18.326(015)$ & $17.472(007)$ \\
\hline $\mathrm{C} 13 \ldots$ & $21.969(371)$ & $20.692(053)$ & $19.294(017)$ & $17.730(010)$ & $21.872(192)$ & $19.940(029)$ \\
\hline $\mathrm{C} 14 \ldots \ldots$ & $17.251(019)$ & $15.996(008)$ & $15.520(008)$ & $15.353(008)$ & $16.371(009)$ & $15.709(007)$ \\
\hline
\end{tabular}

NoTE.-Uncertainties given in parentheses in thousandths of a magnitude correspond to the rms of the magnitudes obtained on four photometric nights, with a minimum uncertainty of 0.015 mag for an individual measurement. 
TABLE 2

$u^{\prime} g^{\prime} r^{\prime} i^{\prime} B V$ PнотометRY of SN 2005 bf

\begin{tabular}{|c|c|c|c|c|c|c|c|c|c|c|c|}
\hline JD $-2,453,000$ & $\begin{array}{l}\text { Epoch }^{\mathrm{a}} \\
\text { (days) }\end{array}$ & Telescope & $u^{\prime}$ & $g^{\prime}$ & $r^{\prime}$ & $i^{\prime}$ & $B$ & V & $\begin{array}{l}T_{\mathrm{bb}} \\
(\mathrm{K})\end{array}$ & $\begin{array}{l}\log L_{\left(u^{\prime} \rightarrow i^{\prime}\right)} \\
\left(\mathrm{ergs} \mathrm{s}^{-1}\right)\end{array}$ & $\begin{array}{r}\log L_{\text {bol }} \\
\left(\mathrm{ergs} \mathrm{s}^{-1}\right)\end{array}$ \\
\hline $444.81 \ldots \ldots \ldots \ldots . . .$. & -54 & KAIT & $\ldots$ & $\ldots$ & $>20.0$ & & & & . & & \\
\hline $466.80 \ldots \ldots \ldots \ldots \ldots$ & -32 & KAIT & $\ldots$ & $\ldots$ & $18.070(062)$ & $\ldots$ & & $\ldots$ & $\ldots$ & & $(42.100)$ \\
\hline $467.65 \ldots \ldots \ldots \ldots \ldots$ & -32 & Swope & $18.691(021)$ & $17.991(015)$ & $17.935(015)$ & $18.048(015)$ & $18.191(016)$ & $17.962(015)$ & 9567 & 42.014 & 42.146 \\
\hline 468.58. & -31 & Swope & $18.436(016)$ & $17.891(015)$ & $17.881(015)$ & $18.006(017)$ & $18.086(016)$ & $17.894(015)$ & 10082 & 42.061 & 42.183 \\
\hline $474.57 \ldots$ & -25 & Swope & $17.939(016)$ & $17.478(015)$ & $17.462(015)$ & $17.526(015)$ & $17.686(016)$ & $17.464(015)$ & 9575 & & 42.365 \\
\hline $475.64 \ldots \ldots$ & -24 & Swope & $17.994(016)$ & $17.484(015)$ & 17.409 & $17.481(015)$ & $17.678(016)$ & $17.451(015)$ & 9214 & & 42.373 \\
\hline & -24 & KAIT & & & 17.419 & & & & & & \\
\hline & -23 & Swope & $18.092(016)$ & $17.510(015)$ & $17.406(015)$ & $17.477(015)$ & $17.721(016)$ & $17.460(015)$ & 8912 & 42.223 & 42.365 \\
\hline $476.73 \ldots$ & -23 & KAIT & & $\ldots$ & $17.426(033)$ & $\ldots$ & & & & & \\
\hline $477.56 \ldots \ldots \ldots . .$. & -22 & Swope & $18.244(016)$ & $17.543(015)$ & $17.371(015)$ & $17.414(015)$ & $17.762(016)$ & $17.475(015)$ & 297 & 42.209 & 2.366 \\
\hline $478.57 \ldots \ldots \ldots \ldots \ldots$ & -21 & Swope & $18.348(017)$ & $17.554(015)$ & $17.344 \mathrm{~d}$ & $17.392(015)$ & $17.806(016)$ & 17.457 & 974 & & 2.366 \\
\hline $483.54 \ldots \ldots$ & -16 & Swope & $18.530(037)$ & $17.370(015)$ & $17.113(015)$ & $17.148(015)$ & $17.646(016)$ & $17.223(015)$ & 7596 & & 42.442 \\
\hline $484.61 \ldots \ldots \ldots \ldots \ldots$ & -15 & Swope & $18.369(041)$ & $17.314(015)$ & $17.060(015)$ & $17.082(015)$ & $17.616(016)$ & $17.166(015)$ & 7485 & & 42.469 \\
\hline $489.51 \ldots \ldots \ldots \ldots \ldots$ & -10 & Swope & $17.871(016)$ & $16.905(015)$ & $16.704(015)$ & $16.746(015)$ & $17.148(016)$ & $16.808(015)$ & 8033 & & 42.617 \\
\hline $490.50 \ldots \ldots$. & -9 & Swope & $17.681(016)$ & $16.787(015)$ & $16.622(015)$ & $16.688(015)$ & $17.029(016)$ & $16.722(015)$ & 8350 & & 42.652 \\
\hline $491.48 \ldots$ & -8 & Swope & $17.506(016)$ & $16.694(015)$ & $16.552(015)$ & $16.645(017)$ & $16.923(016)$ & 16.630 & 8675 & & 42.683 \\
\hline 493.51 & -6 & Swope & $17.273(043)$ & $16.603(015)$ & $\ldots$ & $\ldots$ & 16.799 ( & 16.532 & 8677 & & 42.734 \\
\hline $495.53 \ldots$. & -4 & Swope & $17.147(016)$ & 16.528( & . & & 16.754 & 16.477 & 8486 & & 42.768 \\
\hline $496.54 \ldots \ldots \ldots \ldots . .$. & -3 & Swope & $17.145(017)$ & 16.501( & & & 16.724 & 16.472 & 19 & & 2.778 \\
\hline $497.46 \ldots \ldots \ldots \ldots \ldots$ & -2 & Swope & $17.097(016)$ & $16.493(015)$ & $\ldots$ & $\ldots$ & $16.697(016)$ & $16.448(015)$ & 8333 & 42.635 & 42.793 \\
\hline $497.47 \ldots$. & -2 & Clay & & & $16.348(015)$ & $16.347(030)^{\mathrm{b}}$ & & & & & \\
\hline $499.45 \ldots$ & 0 & Swope & $17.105(016)$ & $16.495(015)$ & & & $16.730(016)$ & $16.432(015)$ & 8051 & 42 & 42.799 \\
\hline $500.46 \ldots$ & 1 & Swope & $17.142(016)$ & $16.529(015)$ & & & 16.743 & 16.460 & 3 & & .798 \\
\hline $501.46 \ldots \ldots$ & 2 & Swope & $17.198(016)$ & $16.559(0$ & & & $16.801(016)$ & 16.460( & 7601 & & 794 \\
\hline 51 & 13 & Swope & $18.313(062)$ & $17.123(015)$ & $16.579(015)$ & $16.441(015)$ & 17.561 & 16.8 & 5719 & 4 & 2.682 \\
\hline $46 \ldots$ & 13 & Swope & & $\ldots$ & $16.626(015)$ & $16.490(015)$ & $17.570(016)$ & $16.881(015)$ & 5776 & 87 & 42.662 \\
\hline $516.45 \ldots$. & 16 & Swope & $18.695(024)$ & $17.426(015)$ & $\ldots$ & $\ldots$ & $17.863(016)$ & $17.052(015)$ & 5383 & 42.304 & 42.614 \\
\hline $517.45 \ldots \ldots \ldots \ldots \ldots$ & 17 & Swope & $18.792(071)$ & $17.513(029)$ & $\ldots$ & $\ldots$ & $17.992(016)$ & $17.127(015)$ & 5240 & 42.273 & 42.596 \\
\hline $520.46 \ldots \ldots \ldots \ldots$ & 20 & Swope & $19.271(035)$ & $17.801(015)$ & $\ldots$ & $\ldots$ & $18.324(016)$ & $17.367(015)$ & 4945 & 42.169 & 42.529 \\
\hline $521.44 \ldots \ldots \ldots \ldots \ldots$ & 21 & Swope & $19.361(043)$ & $17.854(022)$ & $\ldots$ & $\ldots$ & $18.399(016)$ & $17.439(015)$ & 4938 & 42.141 & 42.504 \\
\hline $522.45 \ldots \ldots \ldots \ldots$ & 22 & Swope & $\ldots$ & $\ldots$ & $17.195(015)$ & $16.924(015)$ & $18.478(025)$ & $17.535(018)$ & 4879 & 42.109 & 42.480 \\
\hline $523.47 \ldots \ldots \ldots \ldots \ldots$ & 23 & Swope & $\ldots$ & $\ldots$ & $17.263(015)$ & $17.018(015)$ & $18.609(018)$ & $17.641(015)$ & 4821 & 42.070 & 42.446 \\
\hline $524.45 \ldots \ldots \ldots \ldots \ldots$ & 24 & Swope & $\ldots$ & $\ldots$ & $17.377(020)$ & $17.075(027)$ & $18.737(017)$ & $17.697(015)$ & 4750 & 42.034 & 42.423 \\
\hline $525.45 \ldots \ldots \ldots \ldots \ldots$ & 25 & Swope & $\ldots$ & $\ldots$ & $17.421(015)$ & $17.127(015)$ & $18.811(038)$ & $17.773(027)$ & 4698 & 42.007 & 42.403 \\
\hline $526.45 \ldots \ldots \ldots \ldots \ldots$ & 26 & Swope & $\cdots$ & $\ldots$ & $17.458(015)$ & $17.201(015)$ & $18.975(051)$ & $17.878(018)$ & 4575 & 41.974 & 42.381 \\
\hline $527.51 \ldots$ & 27 & Swope & $\cdots$ & $\ldots$ & $17.540(015)$ & $17.313(015)$ & $18.987(016)$ & $17.953(015)$ & 4688 & 41.942 & 42.333 \\
\hline $528.47 \ldots$ & 28 & Swope & & $\ldots$ & $\ldots$ & $\ldots$ & $18.999(016)$ & $18.003(015)$ & 4737 & 41.920 & 42.305 \\
\hline $529.48 \ldots$ & 29 & Swope & $20.260(046)$ & $18.535(015)$ & & & $19.097(017)$ & $18.061(015)$ & 4713 & 41.891 & 42.275 \\
\hline $530.47 \ldots \ldots$ & 30 & Swope & $20.274(039)$ & $18.588(021)$ & & & $19.167(018)$ & $18.105(017)$ & 4729 & 41.870 & 42.245 \\
\hline $538.48 \ldots \ldots \ldots \ldots \ldots$ & 38 & Swope & $\ldots$ & $\ldots$ & $\cdots$ & & $19.520(037)$ & $18.464(015)$ & & & (42.124) \\
\hline $538.49 \ldots \ldots \ldots \ldots$ & 38 & Swope & $\ldots$ & & & & $19.583(043)$ & $18.472(015)$ & & & $(42.135)$ \\
\hline $539.46 \ldots \ldots \ldots \ldots \ldots$ & 39 & Swope & $\ldots$ & $\cdots$ & & & & $18.484(019)$ & $\cdots$ & & $\ldots$ \\
\hline $542.46 \ldots \ldots \ldots \ldots$ & 42 & Swope & $\ldots$ & $\ldots$ & $\ldots$ & $\ldots$ & $19.776(068)$ & $18.598(018)$ & $\ldots$ & $\cdots$ & (42.103) \\
\hline $565.47 \ldots \ldots \ldots \ldots \ldots$ & 64 & Swope & $\ldots$ & $\ldots$ & $9.061(416)$ & $18.715(177)$ & $20.403(063)$ & $19.357(022)$ & 4685 & 41.279 & 41.734 \\
\hline $569.47 \ldots \ldots \ldots \ldots \ldots$ & 68 & Swope & $\ldots$ & $\ldots$ & $\ldots$ & $\ldots$ & $20.625(133)$ & $19.582(042)$ & 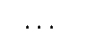 & 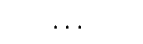 & (41.673) \\
\hline
\end{tabular}

NotE.-Photometric uncertainties given in parentheses in thousandths of a magnitude. A minimum uncertainty of 0.015 mag was set.

${ }^{a}$ Rest-frame days since $L_{\text {bol }}$ maximum (JD 2,453,499.8).

${ }^{\mathrm{b}}$ Synthetic magnitude computed from the spectrum obtained the same night. 


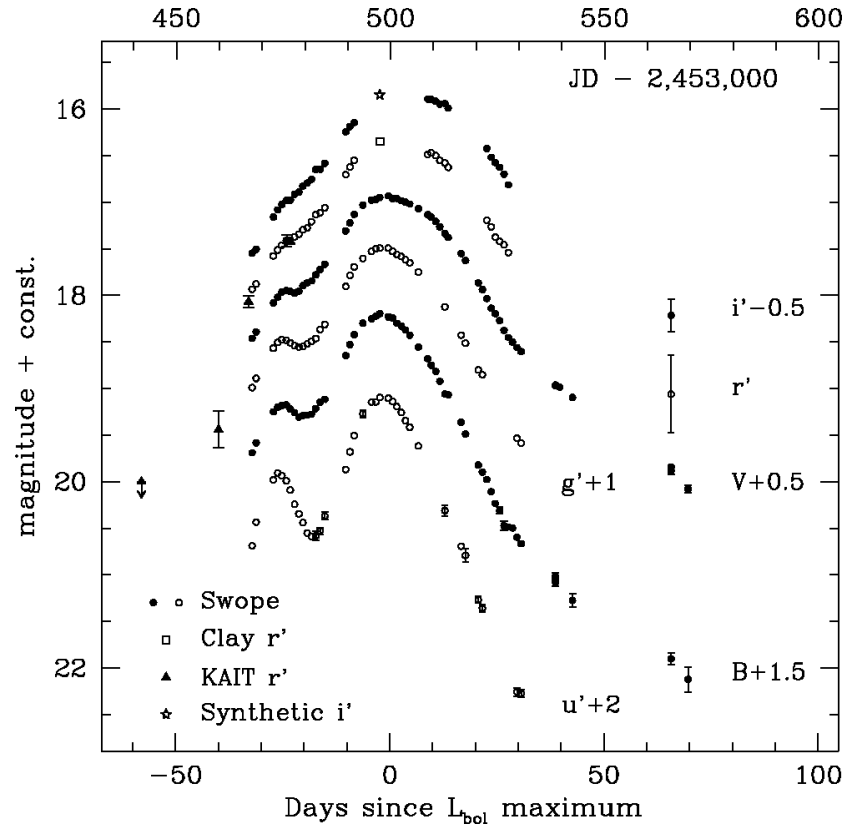

FIG. 2.-Observed $u^{\prime} g^{\prime} r^{\prime} i^{\prime} B V$ light curves of SN 2005bf from Swope (open and filled circles), KAIT ( filled triangles), and Clay (open square). A star symbol marks a synthetic $i^{\prime}$ magnitude obtained from the LDSS-3 spectrum of May 7 ( JD 2,453,497). The arrow marks an $r^{\prime}$ lower limit from a KAIT image obtained on March $15(\sim \mathrm{JD} 2,453,445)$. Unless explicitly drawn, the error bars are smaller than the symbols. The time axes are given in the observer's frame. For clarity, the magnitudes in each band have been shifted by an arbitrary constant.

the SN on April 15 (JD 2,453,475; $r^{\prime}=17.419 \mathrm{mag}$ ) and 16 (JD $\left.2,453,476 ; r^{\prime}=17.426 \mathrm{mag}\right)$ with those obtained with the Swope telescope on the same nights $\left(r^{\prime}=17.409\right.$ and 17.406 , respectively) confirms that the effective wavelength of the $r^{\prime}$ filter is a good match to that of the KAIT unfiltered bandpass. The first LOSS observation obtained on March 15 (JD 2,435,445), in which the SN is not detectable, was used to derive a lower limit to the SN magnitude of $r^{\prime}>20.0 \mathrm{mag}$.

A 17 day gap in the $r^{\prime}$ and $i^{\prime}$ observations with the Swope telescope was partially filled with an $r^{\prime}$ image and a spectrum obtained on May 7 (JD 2,453,497) using the Low Dispersion Survey Spectrograph 3 (LDSS-3) at the Clay $6.5 \mathrm{~m}$ telescope at LCO. For this purpose, we used the filter response functions of $r^{\prime}$ and $i^{\prime}$ given by Smith et al. (2002) to compute a synthetic $\left(r^{\prime}-i^{\prime}\right)$ color from the spectrum and thence to derive a synthetic $i^{\prime}$ magnitude from the observed $r^{\prime}$ magnitude.

The resulting $u^{\prime} g^{\prime} r^{\prime} i^{\prime} B V$ magnitudes of SN 2005bf and their uncertainties are listed in Table 2. A minimum uncertainty of 0.015 mag was assumed for a single measurement based on the typical scatter in the transformation from instrumental to standard magnitudes of bright stars (Paper I). Figure 2 shows the corresponding light curves. We computed $K$-corrections for the $g^{\prime} r^{\prime} i^{\prime} B V$ filters (the $u^{\prime}$ passband lies beyond the coverage of our spectra) using the available spectra. Given the small redshift of the $\mathrm{SN}$, the corrections proved small, within $\pm 0.02 \mathrm{mag}$ in all cases. We therefore decided to neglect them in our analysis.

Errors in our photometry could potentially arise from a poor subtraction of underlying host-galaxy light. Fortunately, SN 2005 bf was located in an outer region of the galaxy (see Fig. 1). Inspection of the prediscovery template LOSS images reveals no strong background variations at the location of the SN within a radius of $\sim 3^{\prime \prime}$ or in the sky annulus. If the contamination were large, the seeing changes from epoch to epoch would cause a large variation in such contamination and, consequently, a large scatter
TABLE 3

Light-Curve Parameters for SN 2005bf

\begin{tabular}{|c|c|c|}
\hline Filter & JD at Peak $-2,453,000$ & Peak Magnitude \\
\hline$u^{\prime}$ & $497.9 \pm 0.5$ & $17.10 \pm 0.01$ \\
\hline$g^{\prime}$ & $498.1 \pm 0.5$ & $16.50 \pm 0.01$ \\
\hline$r^{\prime} \ldots$ & $500.2 \pm 1.0$ & $16.32 \pm 0.01$ \\
\hline$i^{\prime}$ & $502.9 \pm 1.0$ & $16.32 \pm 0.02$ \\
\hline (n)............... & $497.4 \pm 0.5$ & $16.71 \pm 0.02$ \\
\hline$V \ldots \ldots \ldots \ldots \ldots \ldots \ldots \ldots \ldots \ldots \ldots \ldots \ldots \ldots$ & $498.8 \pm 0.5$ & $16.44 \pm 0.01$ \\
\hline 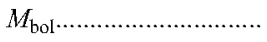 & $499.8 \pm 0.5$ & $-18.32 \pm 0.03^{\mathrm{a}}$ \\
\hline
\end{tabular}

NoTES.- Uncertainties in the peak magnitudes were estimated from the rms of the photometric points about a low-order polynomial fit. Uncertainties in the dates of maximum are based on the data sampling rates.

a Absolute bolometric magnitude based on $L_{\mathrm{bol}}$ from Table 2 (no hostgalaxy extinction correction applied).

in the light curves. The small scatter seen in the light curves (typically $0.01-0.03 \mathrm{mag}$ ) is an indication that the contamination must be very small.

As mentioned in $\S 1$, the $u^{\prime} g^{\prime} B V$ light curves of SN 2005bf show a unique behavior consisting of a first maximum around April 13 (JD 2,453,474), a subsequent decline lasting several days, a second rise that brings the $\mathrm{SN}$ to a main maximum around May 8 (JD 2,453,498), and finally a 30 day fast decline phase followed by a slower decline phase. Although the $r^{\prime} i^{\prime}$ light curves do not show this double maximum, they do show a shoulder around the time of the first maximum. The other remarkable feature is the long time ( $\sim 40$ days) in all filters the SN took to reach the main maximum. There is a large range in light-curve width among Type Ib/Ic SNe (Hamuy 2004), with the hypernovae SN 1997ef and SN 1998bw having two of the broadest ones, but even these objects took between 15 and 20 days to reach maximum light (Galama et al. 1998; Iwamoto et al.

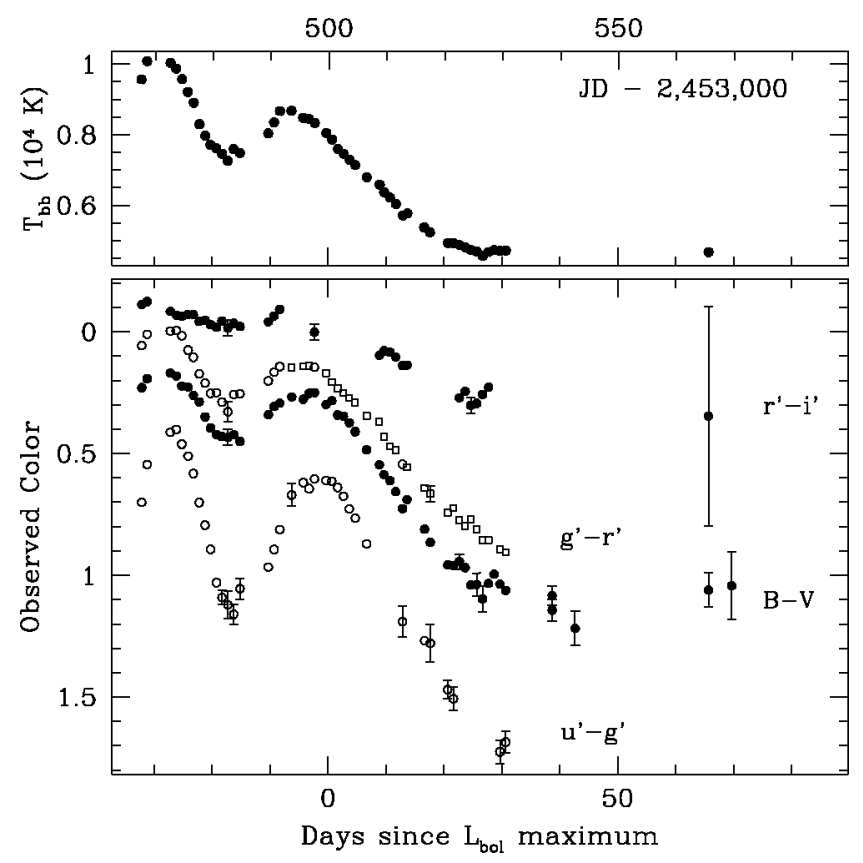

Fig. 3.-Top: Temperature $T_{\mathrm{bb}}$ from the blackbody fits to the $B$ through $i^{\prime}$ monochromatic fluxes. Bottom: Observed color evolution for $\left(u^{\prime}-g^{\prime}\right),(B-V)$, $\left(g^{\prime}-r^{\prime}\right)$, and $\left(r^{\prime}-i^{\prime}\right)$. Circles mark colors computed directly from measurements in both bands, while squares are used when one of the light curves was interpolated in time. Unless explicitly drawn, the error bars are smaller than the symbols. The time axes are given in the observer's frame. No reddening correction was applied. 
TABLE 4

Near-Infrared Photometry of Two Comparison Stars

\begin{tabular}{ccccc}
\hline \hline Star ID & $Y$ & $J$ & $H$ & $K_{s}$ \\
\hline $\mathrm{C} 10 \ldots \ldots \ldots \ldots \ldots$. & $17.40(02)$ & $17.16(02)$ & $16.87(05)$ & $16.72(06)$ \\
$\mathrm{C} 11 \ldots \ldots \ldots \ldots \ldots$. & $16.97(02)$ & $16.58(02)$ & $16.12(02)$ & $16.00(03)$ \\
\hline
\end{tabular}

NoTE.- Uncertainties given in parentheses in hundredths of a magnitude. A minimum uncertainty of $0.02 \mathrm{mag}$ was set.

2000). Table 3 lists the dates of maximum and peak magnitudes for SN 2005bf in $u^{\prime} g^{\prime} r^{\prime} i^{\prime} B V$ and in the bolometric light curve (see $\$ 4.2$ ).

Figure 3 shows the $\mathrm{SN}$ colors along with the temperature derived from blackbody (BB) fits to the $g^{\prime} r^{\prime} i^{\prime} B V$ magnitudes as described in $\S 4.2$. All of the color curves track the temperature evolution very closely. We observe a temperature rise reaching $10,000 \mathrm{~K}$ at the time of the first maximum, a cooling phase for a period of 10 days, which brings the temperature down to $7300 \mathrm{~K}$, a subsequent reheating period of 13 days reaching $8700 \mathrm{~K}$ close to the time of maximum luminosity, followed by a steady second cooling phase past maximum light.

In addition to the optical data, $Y J H K_{s}$ photometry was obtained at three epochs using the Wide Field Infrared Camera (WIRC; Persson et al. 2002) and the Persson Auxiliary Nasmyth Infrared Camera (PANIC; Martini et al. 2004) mounted on the du Pont $2.5 \mathrm{~m}$ and the Baade $6.5 \mathrm{~m}$ telescopes at LCO, respectively. Two comparison stars in the SN field were calibrated using observations of standard stars of Persson et al. (1998) obtained on May 21 (JD 2,453,511) with the Baade $6.5 \mathrm{~m}$ telescope. Table 4 lists the $Y J H K_{s}$ magnitudes for the comparison stars. SN magnitudes were computed differentially relative to the comparison stars with an aperture of $2^{\prime \prime}$ (see Paper I for details). The $Y J H K_{s}$ photometry for SN 2005 bf is listed in Table 5. The quoted uncertainties assume a minimum error of $0.02 \mathrm{mag}$ on an individual measurement (Paper I).

\section{SPECTROSCOPY}

A total of nine optical spectra were obtained using the long-slit grism mode of the Wide Field Reimaging CCD Camera (WFCCD) at the du Pont $2.5 \mathrm{~m}$ telescope and the LDSS-3 (Allington-Smith et al. 1994) at the Clay $6.5 \mathrm{~m}$ telescope, both at LCO. An additional spectrum was obtained with the Low Resolution Imaging Spectrometer (LRIS; Oke et al. 1995) on the Keck I 10 m telescope. Table 6 gives a journal of the spectroscopic observations.

In general, the spectra were obtained using a narrow $\left(\sim 1^{\prime \prime}-2^{\prime \prime}\right)$ slit oriented near the parallactic angle (Filippenko 1982). All of the WFCCD spectra were obtained with a single instrumental setup, without order-blocking filters, and are thus subject to second-order contamination effects at wavelengths longer than $7000 \AA$. The LDSS-3 and LRIS spectra were instead obtained with two instrumental setups (blue and red channels) and using order-blocking filters. The spectrum from May 31 (JD 2,543,521) was obtained through a wider (8.65) slit. All the spectra were wavelength and flux calibrated using arc lamp and flux standard observations (Hamuy et al. 1994). In some cases, a smoothspectrum star selected from Table 4 of Bessell (1999) was observed to correct for telluric absorption features (see Paper I).

In addition, a spectrum of the underlying host galaxy near the location of the SN was extracted from the LDSS-3 observation of May 7 (JD 2,453,497). Several emission ( $\mathrm{H} \alpha$ and $\left[\mathrm{N}_{\text {II }}\right] \lambda \lambda 6548$, 6583) and absorption ( $\mathrm{Na} I \mathrm{D} 1$ and D2 and $\mathrm{Ca} \amalg \mathrm{H}$ and $\mathrm{K}$ ) lines were identified, which allowed us to derive an average recession velocity at the location of the SN of $5496 \pm 27 \mathrm{~km} \mathrm{~s}^{-1}$. This is somewhat lower than the recession velocity of $5670 \pm 8 \mathrm{~km} \mathrm{~s}^{-1}$ given by Falco et al. (1999) for the host galaxy, a difference probably caused by the rotation of the galaxy. The former velocity was adopted to shift the observed wavelength axis of the spectra to the SN rest frame.

Figure 4 shows the series of SN spectra sorted in time. (The LDSS-3 spectrum on JD 2,453,502.48 is excluded, to avoid crowding; it spans only $6057-10000 \AA$, in any case.) The main spectral features are labeled with the ions that produce them. The positions of the two main telluric absorption features are marked with an Earth symbol. The numbers in parenthesis give the epoch of each spectrum in SN rest-frame days since the time of maximum bolometric luminosity (JD 2,453,499.8; see $\S 4.2$ ). We hereafter use these epochs to label the spectra.

\section{ANALYSIS}

\subsection{Spectroscopic Analysis}

SN 2005bf was classified by Morrell et al. (2005) as a Type Ic event due to the absence of strong $\mathrm{H}_{\mathrm{I}}$ and $\mathrm{He}_{\mathrm{I}}$ lines, which are the distinguishing features of Type II and Ib SNe (e.g., Filippenko 1997), respectively. Although the initial spectra of SN 2005bf showed a prominent absorption at $\sim 6250 \AA$ that could be attributed to $\mathrm{Si}$ II $\lambda 6355$, it was not nearly as strong as that observed in typical Type Ia SNe, nor was there any evidence in the spectra for the $S_{\text {II }} \lambda \lambda 5454,5640$ feature, which is also prominent around maximum light in Type Ia SNe. The two topmost spectra shown in Figure 5 compare SN 2005bf(day -32) and the Type Ic SN 1994I 5 days before maximum light (Filippenko et al. 1995). The comparison shows that at early times, SN 2005 bf could be classified as a Type Ic SN. A similar comparison of SN 2005bf with an early-time spectrum of SN 1987M led Modjaz et al. (2005) to independently classify SN 2005 bf as a Type Ic event. As SN 2005 bf evolved, however, the He I lines grew in strength, and as

TABLE 5

Near-Infrared Photometry of SN 2005bf

\begin{tabular}{|c|c|c|c|c|c|c|}
\hline JD $-2,453,000$ & $\begin{array}{c}\text { Epoch }^{\mathrm{a}} \\
\text { (days) }\end{array}$ & $Y$ & $J$ & $H$ & $K_{S}$ & Instrument \\
\hline$\ldots \ldots \ldots \ldots \ldots \ldots \ldots$ & -16 & $16.93(03)$ & $16.75(03)$ & $16.65(05)$ & $\ldots$ & WIRC \\
\hline 483.52 & -16 & $16.92(03)$ & $16.76(03)$ & $16.64(05)$ & $\ldots$ & WIRC \\
\hline $511.46 \ldots \ldots \ldots \ldots \ldots \ldots \ldots \ldots \ldots \ldots \ldots \ldots \ldots \ldots$ & 11 & $16.19(03)$ & $15.95(03)$ & $15.87(05)$ & $15.80(06)$ & PANIC \\
\hline $538.46 \ldots \ldots \ldots \ldots \ldots \ldots \ldots \ldots \ldots \ldots$ & 38 & $\ldots$ & $16.91(03)$ & $\ldots$ & $16.43(05)$ & PANIC \\
\hline
\end{tabular}

NoTES. - WIRC data were obtained with two detectors and their magnitudes agree within uncertainties. We use the averages in the present analysis. Uncertainties are given in parentheses in hundredths of a magnitude. A minimum uncertainty of $0.02 \mathrm{mag}$ was set for individual measurements. Uncertainties in the zero points arising from uncertainties in the magnitudes of comparison stars were added in quadrature to the measurement errors.

${ }^{a}$ Rest-frame days since $L_{\text {bol }}$ maximum (JD 2,453,499.8). 
TABLE 6

SPECTROSCOPIC ObSERVATIONS OF SN 2005bf

\begin{tabular}{|c|c|c|c|c|c|c|}
\hline JD $-2,453,000$ & $\begin{array}{r}\text { Epoch }^{\mathrm{a}} \\
\text { (days) }\end{array}$ & Instrument & $\begin{array}{l}\text { Wavelength Range } \\
\qquad(\AA)\end{array}$ & $\begin{array}{c}\text { Resolution }^{\mathrm{b}} \\
(\AA)\end{array}$ & $\begin{array}{l}\text { Exposure } \\
\text { (s) }\end{array}$ & $\begin{array}{c}\langle\mathrm{S} / \mathrm{N}\rangle^{\mathrm{c}} \\
(\text { in } 10 \AA)\end{array}$ \\
\hline 467.57. & -32 & WFCCD & $3800-9235$ & 8 & 900 & 69 \\
\hline …… & -27 & WFCCD & $3800-9235$ & 8 & 900 & 88 \\
\hline $475.63 \ldots \ldots \ldots$ & -24 & WFCCD & $3800-9235$ & 8 & 900 & 74 \\
\hline $479.51 \ldots$ & -20 & WFCCD & $3800-9235$ & 8 & 700 & 32 \\
\hline $497.47 \ldots \ldots \ldots \ldots$ & -2 & LDSS-3 & $3838-10000$ & $4^{\mathrm{d}}$ & 200 & 262 \\
\hline $501.89 \ldots \ldots$ & 2 & LRIS & $3100-9350$ & 4 & $300 / 360^{\mathrm{e}}$ & 343 \\
\hline $502.48 \ldots \ldots \ldots$ & 3 & LDSS-3 & $6057-10000$ & 5 & 900 & 200 \\
\hline $521.50 \ldots \ldots \ldots$ & 21 & WFCCD & $3800-8125$ & $8^{\mathrm{f}}$ & 1200 & 64 \\
\hline $523.49 \ldots \ldots \ldots \ldots \ldots \ldots \ldots$ & 23 & WFCCD & $3800-8128$ & 8 & 1200 & 42 \\
\hline $527.54 \ldots \ldots$ & 27 & WFCCD & $3800-8128$ & 8 & 600 & 55 \\
\hline
\end{tabular}

Note.- Some spectra are the combination of multiple observations. In those cases, total exposure times are given.

${ }^{a}$ Rest-frame days since $L_{\text {bol }}$ maximum (JD 2,453,499.8).

b Average resolution obtained from the FWHM of arc lamp lines.

c Average $\mathrm{S} / \mathrm{N}$ in $10 \AA$ bins calculated in the range from 4000 to $8000 \AA$.

${ }^{\mathrm{d}}$ Resolution of $2.9 \AA$ in the blue channel $(3800<\lambda<6000 \AA)$, and $4.7 \AA$ in the red channel $(6000<\lambda<10000 \AA)$.

${ }^{e}$ Different exposure times in blue channel (300 s) and red channel (360 s).

${ }^{\mathrm{f}}$ A wide slit (8"65) was used. In this case the resolution was estimated from the FWHM of the spatial profile of the SN.

shown at the bottom of Figure 5, the spectra from day +21 and later became almost identical to those of the Type Ib SN 1984L. This transformation was also noticed by Wang \& Baade (2005). Interestingly, as shown in the middle of Figure 5 , the spectrum from day -20 (and also those from days -2 and +2 ) was remarkably similar to spectra of the intermediate Type $\mathrm{Ib} / \mathrm{Ic} \mathrm{SN}$ 1999ex obtained around maximum light (Hamuy et al. 2002).

Figure 4 indicates that the $\mathrm{He}$ I $\lambda \lambda 5876,6678,7065$ lines were present, albeit weakly, in the first spectra of SN $2005 \mathrm{bf}$ and grew

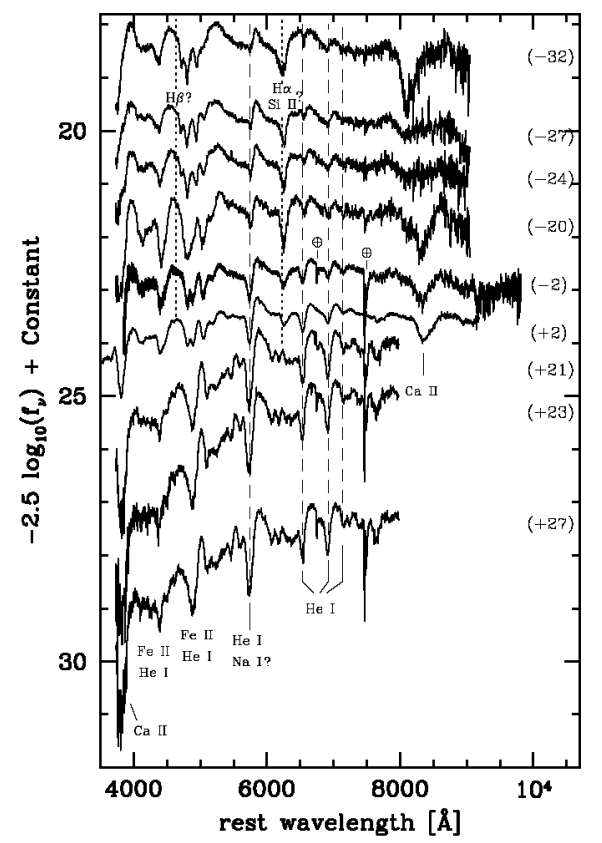

FIG. 4.-Spectroscopic evolution of SN 2005bf. Each spectrum is presented on a logarithmic scale, shifted by an arbitrary constant. The wavelength of the spectra was shifted to the $\mathrm{SN}$ rest frame using a redshift of $z=0.01833$. The labels in parentheses to the right of each spectrum indicate the epoch in restframe days since $L_{\mathrm{bol}}$ maximum ( $\mathrm{JD}=2,453,499.8$ ). The spectrum from day -20 was smoothed by averaging over 5 pixels. The name of the ions responsible for some of the main features are given. The dotted vertical lines mark the wavelength at which the minimum of the $\mathrm{H} \alpha$ and $\mathrm{H} \beta$ lines would appear if hydrogen were present at $\sim 14,500 \mathrm{~km} \mathrm{~s}^{-1}$. The dashed vertical lines mark the absorptions due to $\mathrm{He}$ I $\lambda \lambda 5876,6678,7065,7281$, at an expansion velocity of $\sim 6000 \mathrm{~km} \mathrm{~s}^{-1}$. Telluric features are marked with an Earth symbol $(\oplus)$. steadily in strength during the period covered by our observations. The expansion velocities as measured from the absorption minima of these features were nearly constant, at a value of $\sim 8000-10,000 \mathrm{~km} \mathrm{~s}^{-1}$, until maximum light and dropped to $\sim 6000 \mathrm{~km} \mathrm{~s}^{-1}$ after that.

As a tool for analyzing the premaximum spectral evolution of SN 2005bf, we used the SYNOW code (Fisher 2000) to calculate synthetic spectral fits to our first five spectra. Following the precepts of Branch et al. (2002), we assumed a power-law radial density gradient of index $n=8$ and a Boltzmann excitation temperature of $T_{\mathrm{exc}}=7000 \mathrm{~K}$ for all of these fits. In general, only the $\mathrm{Fe}$ II, $\mathrm{Ca}$ II, $\mathrm{He}$ I, and $\mathrm{H}$ I ions were included in the

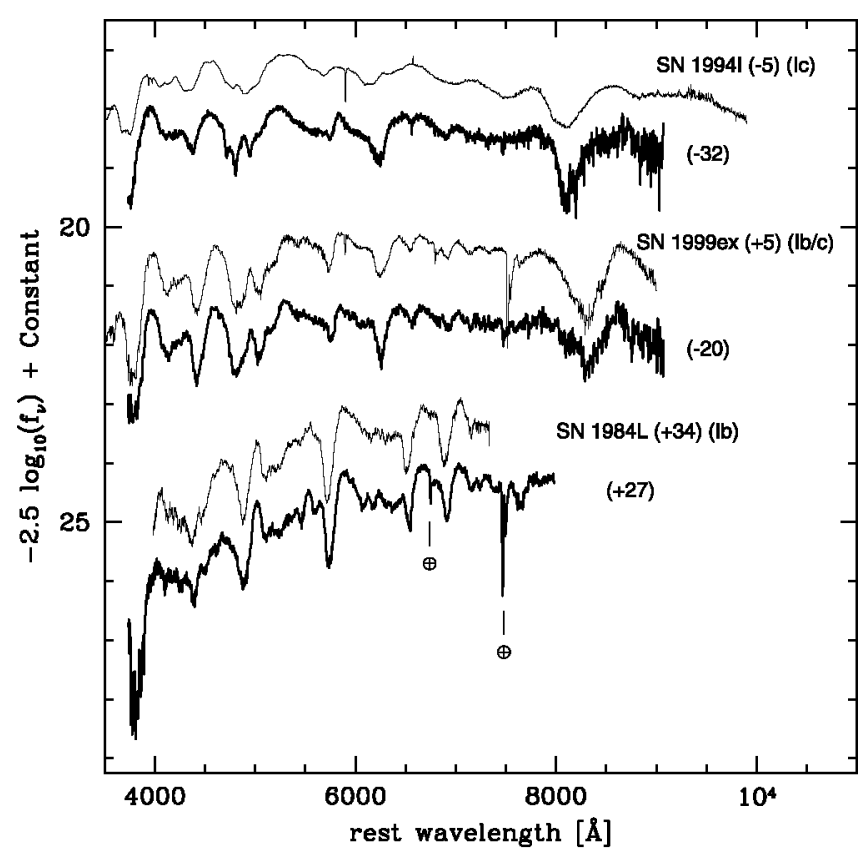

FIG. 5.-Comparison of spectra of SN 2005bf with those of other Type Ib and Ic $\mathrm{SNe}$. At the top, the spectrum from day -32 compared with that of the Type Ic SN 1994I 5 days before maximum light (Filippenko et al. 1995). At the middle, the spectrum from day -20 and that of the Type Ib/Ic SN 1999ex 5 days after maximum light (Hamuy et al. 2002). At the bottom, the spectrum from day +27 and that of the Type Ib SN 1984L 34 days after maximum light (Harkness et al. 1987). Telluric features are marked with an Earth symbol. 


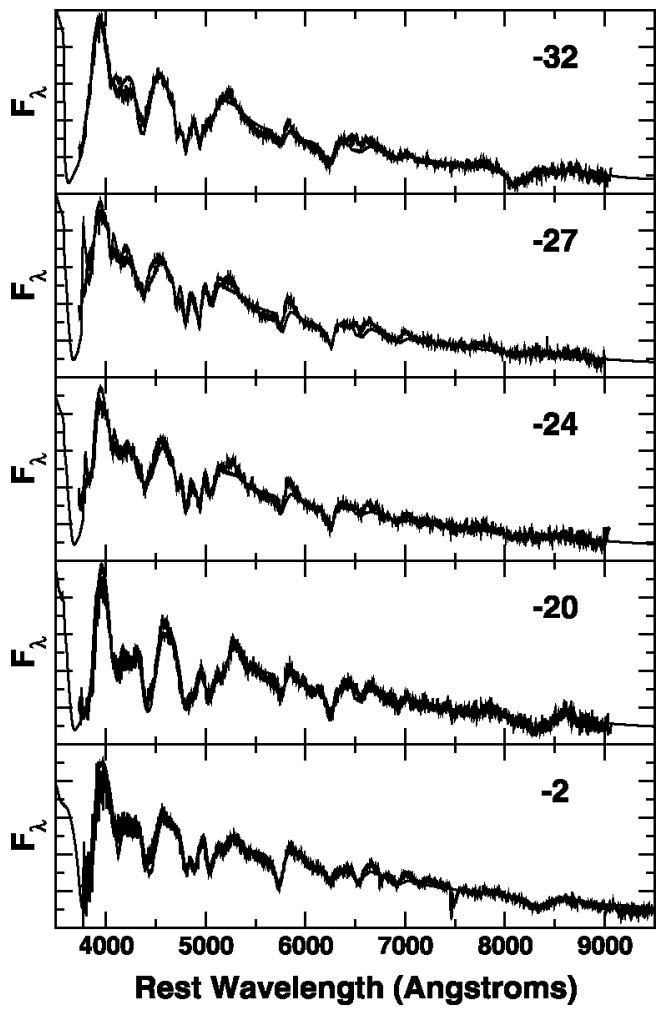

FIG. 6.-SYNOW fits to the premaximum spectra of SN 2005bf. The SYNOW synthetic spectra, which are described in the text, are overplotted on the observed spectra. The epoch is given in rest-frame days relative to $L_{\text {bol }}$ maximum.

calculations. The fits were not intended to reproduce all of the observed features but to provide support to the present analysis. The addition of other ions would not modify our conclusions. Figure 6 shows the final SYNOW fits overplotted on the observed spectra.

With the aid of the SYNOW fits we noticed that the conversion from a Type Ic to a Type Ib SN was accompanied by a sudden change in the expansion velocity of the absorption components of the Fe II $\lambda \lambda 4924,5018,5169$ lines and the Ca II $\lambda \lambda 8498,8542$, 8662 triplet feature. The left half of Figure 7 shows that two separate components of the Fe II lines were present in the spectrum from day -24 : one at an expansion velocity of $\sim 14,000 \mathrm{~km}$ $\mathrm{s}^{-1}$ and the other at $\sim 8000 \mathrm{~km} \mathrm{~s}^{-1}$. In fact, low-velocity and high-velocity components of $\mathrm{Fe}$ II and $\mathrm{Ca}$ II were identifiable as early as our first spectrum (day -32) with the aid of the SYNOW fits. At early epochs, a low-velocity $\mathrm{Ca}$ II component, weaker than its high-velocity counterpart, helps to fit the red wing of the $\mathrm{H}$ and $\mathrm{K}$ absorption. Similarly, a low-velocity Fe II component added to the high-velocity one improves the fit in the region between 4500 and $5200 \AA$. The high-velocity component dominated the spectrum obtained on day -32 but had disappeared by day -2 . The spectra from day -2 onward showed exclusively the lowvelocity absorption component of $\mathrm{Fe}$ II and $\mathrm{Ca}$ II. This strongly suggests that the appearance of high-velocity Fe II and Ca II lines is associated with the initial maximum exhibited by the $u^{\prime} g^{\prime} B V$ light curves. The evolution of the expansion velocities of these components is summarized in the right half of Figure 7.

During the period covered by our spectroscopic observations, we find that the low-velocity component of $\mathrm{Fe}$ II and $\mathrm{Ca}$ II decreased roughly monotonically between $\sim 10,000$ and $\sim 6000 \mathrm{~km}$ $\mathrm{s}^{-1}$. We have identified this with the photospheric velocity in our SYNOW fits. Assuming this interpretation is correct and that the expansion was spherically symmetric, the He I lines are most consistent with arising from a shell that has a maximum velocity of $\sim 11,000 \mathrm{~km} \mathrm{~s}^{-1}$, whereas the high-velocity Fe п, Ca п, and $\mathrm{H} \alpha$ lines are produced in a detached shell at velocities $\geq 14,000 \mathrm{~km} \mathrm{~s}^{-1}$.

The $6250 \AA$ absorption, like the high-velocity Fe II and Ca II lines, was present at early epochs but disappeared by the time of maximum light. A similar feature has been seen in several Type Ib $\mathrm{SNe}$, and its identification is a long-debated issue (e.g., see Wheeler et al. 1994; Branch et al. 2002). We consider the most likely identification to be high-velocity $\left(\sim 15,000 \mathrm{~km} \mathrm{~s}^{-1}\right) \mathrm{H} \alpha$ for two basic reasons: (1) the coincidence in expansion velocity with the $\mathrm{Fe}$ II and $\mathrm{Ca}$ II lines, which show a similar association with the peculiar initial maximum in the $u^{\prime} g^{\prime} B V$ light curves, and (2) the presence of a very weak absorption feature in the spectra from days -24 and -20 (Fig. 4, dotted line), which could be identified as $\mathrm{H} \beta$ at the same expansion velocity and with a strength consistent with that predicted by SYNOW spectra. ${ }^{13}$ We reject the alternative identification of this feature with $\mathrm{Si}$ II $\lambda 6355$, since it would imply an expansion velocity of $\sim 4800 \mathrm{~km} \mathrm{~s}^{-1}$, which is significantly lower than the photospheric velocity estimated at these early epochs. ${ }^{14}$ We consider identifications with C II $\lambda 6580$ or Ne I $\lambda 6402$ unlikely for the same reasons given by Branch et al. (2002) for ordinary Type $\mathrm{Ib} \mathrm{SNe}$. Thus, a small amount of hydrogen appears to be present in the outer ejecta of SN $2005 \mathrm{bf}$.

\subsection{Bolometric Light Curve}

The $u^{\prime} g^{\prime} r^{\prime} i^{\prime} B V$ photometry was used to compute a quasibolometric light curve covering the wavelength range longward of $3000 \AA$. Our broadband magnitudes were corrected for Galactic extinction using $E(B-V)_{\text {Gal }}=0.045 \mathrm{mag}$ (Schlegel et al. 1998 ) and the reddening law with $R_{V}=3.1$ given by Cardelli et al. (1989). Additional extinction originating in the host galaxy is difficult to estimate. Examination of our spectra from days -2 and +2 , which have the best wavelength resolution and signal-tonoise ratios, reveals the presence of interstellar absorption lines of $\mathrm{NaID} 1$ and $\mathrm{D} 2$ and $\mathrm{Ca} \amalg \mathrm{H}$ and $\mathrm{K}$ at the redshift of the host galaxy, suggesting that some extinction exists. However, the uncertainties in estimating $E(B-V)_{\text {Host }}$ from the equivalent widths (EWs) of these lines are large. We measure the total EW of the Na I D 1 and D2 lines to be $\sim 1 \AA$. According to Turatto et al. (2003), two values of the color excess would be favored: $E(B-V)_{\text {Host }} \approx 0.1$ and $0.5 \mathrm{mag}$, depending on the gas-to-dust ratio of the host galaxy environment. We consider the value $E(B-V)_{\text {Host }} \approx 0.5 \mathrm{mag}$ to be unrealistic because it would imply color temperatures $\gtrsim 40,000 \mathrm{~K}$ during the time of initial maximum (see below). Such high temperatures do not agree with the ionic species observed in our spectra. Hence, the lower value of $E(B-V)_{\text {Host }} \approx 0.1$ mag seems more likely. Close examination of the day -2 spectrum reveals $\mathrm{Na}$ I $\mathrm{D}$ absorption due to our own Galaxy at an equivalent width of approximately $\sim 0.5 \AA$. If the interstellar medium in the host galaxy of SN $2005 \mathrm{bf}$ is similar to that of the Galaxy, this would also suggest an extinction of $E(B-V)_{\text {Host }} \approx 0.1$ mag. Given the uncertainties, we adopt $E(B-V)_{\text {Host }}=0.0 \mathrm{mag}$ for our analysis but present the case of $E(B-V)_{\text {Host }}=0.1$ mag as an alternative.

The extinction corrected $u^{\prime} g^{\prime} r^{\prime} i^{\prime} B V$ broadband magnitudes were converted into monochromatic fluxes at the effective wavelengths of $3557,4825,6261,7672,4448$, and $5505 \AA$, respectively,

\footnotetext{
13 According to Wang \& Baade (2005), weak absorption consistent with $\mathrm{H} \beta$ and $\mathrm{H} \gamma$ was also present in a spectrum obtained on April 30 (day -9).

14 Nevertheless, we cannot rule out that the absorption is produced by a blend of high-velocity $\mathrm{H} \alpha$ with Si п $\lambda 6355$ at $8000-10,000 \mathrm{~km} \mathrm{~s}^{-1}$.
} 

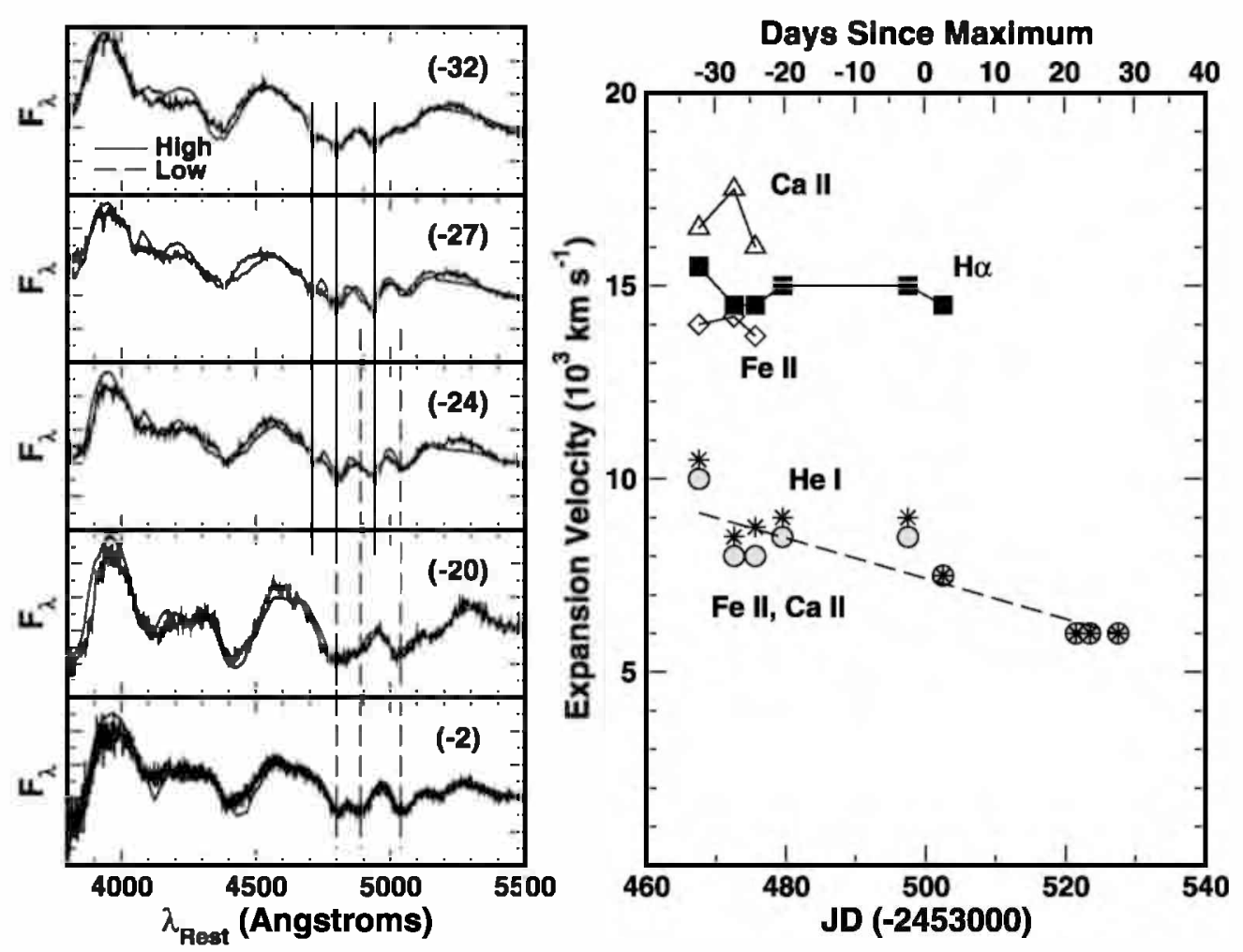

Fig. 7.-Left: Evolution of the spectral region centered on the Fe II $\lambda \lambda 4924,5018,5169$ lines during the rise to maximum light. The observed spectra are compared with SYNOW synthetic spectral calculations. The three vertical solid lines indicate the approximate wavelengths of this Fe $\mathrm{I}$ multiplet in the high-velocity gas that is visible during the peculiar initial maximum in the $u^{\prime} g^{t} B V$ light curves. The three vertical dashed lines show the same multiplet at lower expansion velocity. Note that from day -27 through day -20 , both velocity components are clearly present in the spectra. Right: Expansion velocity measurements for SN 2005bf. Values were estimated from SYNOW analyses of the observed spectra and have typical errors of 500-1000 $\mathrm{km} \mathrm{s}^{-1}$. The diamonds, triangles, and squares correspond to the high-velocity $\mathrm{Fe}$ II, $\mathrm{Ca}$ II, and $\mathrm{H} \alpha$ absorption, respectively, whereas the circles show the lower velocity $\mathrm{Fe}$ II and $\mathrm{Ca}$ II that we associate with the photosphere. The expansion velocities of the He I lines are plotted with an asterisk. The time axes are given in the observer's frame.

as given by Fukugita et al. (1996; their Tables $2 a$ and $2 c$ ). At epochs when a certain filter observation was not available, its magnitude was interpolated in time from the light curve using the surrounding points and a low-order polynomial. The total "UVOIR" flux $F_{u^{\prime}-l^{\prime}}$ in the region between the effective wavelengths of the $u^{\prime}$ and $i^{\prime}$ filters was integrated using the trapezoid approximation. On the blue side of $3557 \AA$ the flux $F_{\nu}(3557)$ derived from the $u^{\prime}$ magnitude was extrapolated with a straight line to zero flux at $3000 \AA$ (the blueward limit of the $u^{\prime}$ filter), and no significant flux was supposed to be emitted at shorter wavelengths, an assumption based on observations of other Type $\mathrm{Ib} / \mathrm{Ic}$ SNe and of SN 1987A (Panagia 2003). On the infrared side the flux was extrapolated to $i=\infty$ using a BB model obtained by fitting the $g^{\prime} r^{\prime} i^{\prime} B V$ fluxes with a Planck function (shifted to the rest frame of the SN ). ${ }^{15}$ The integrated flux under the fitted Planck function between the effective wavelength of the $i^{\prime}$ filter and $i=\infty$ was taken as the IR correction. This correction remained below $35 \%$ of the total flux until a few days after maximum light and increased to $60 \%$ thereafter. A by-product of this procedure is the color temperature $\left(T_{\mathrm{bb}}\right)$ obtained from the BB fits. The values of $T_{\mathrm{bb}}$ for $E(B-V)_{\text {Host }}=0.0$ mag are given in Table 2

\footnotetext{
${ }^{15}$ The $u^{\prime}$ points were excluded from the BB fits because they clearly departed from the model, especially before maximum light. The $u^{\prime}$ fluxes generally lay below the fitted BB curves, probably due to strong line blanketing in that part of the spectrum. The effect of including $u^{\prime}$ would be to lower the derived $\mathrm{BB}$ temperature values. For consistency, we kept the same policy for postmaximum epochs, even though there was a better agreement of the $u^{\prime}$ fluxes with the fitted BB curves.
}

and shown in Figure 3, along with the observed SN colors. ${ }^{16}$ The sum of $F_{u^{t}-i^{\prime}}$ and the UV and IR corrections yielded the bolometric flux $F_{\text {bol }}$.

Both $F_{u^{\prime} \rightarrow i^{\prime}}$ and $F_{\text {bol }}$ were then transformed into $\operatorname{UVOIR}\left(L_{u^{\prime}-i^{\prime}}\right)$ and bolometric luminosity $\left(L_{\text {bol }}\right)$, respectively. We assumed spherical symmetry and used a distance of $83.8 \pm 10.2 \mathrm{Mpc}$ based on the Hubble law, a value of the Hubble constant of $H_{0}=72 \pm$ $8 \mathrm{~km} \mathrm{~s}^{-1} \mathrm{Mpc}^{-1}$ (Freedman et al. 2001), and a recession velocity in the cosmic microwave background frame of $6032 \pm$ $300 \mathrm{~km} \mathrm{~s}^{-1}$ (obtained from the heliocentric velocity given by Falco et al. [1999], and using the velocity transformation tool from the NASA/IPAC Extragalactic Database). The resulting luminosities computed with $E(B-V)_{\text {Host }}=0.0$ mag are listed in Table 2, and the bolometric light curves of SN $2005 \mathrm{bf}$ are shown in Figure 8 for both $E(B-V)_{\text {Host }}=0.0$ mag ( filled circles $)$ and $E(B-V)_{\text {Host }}=0.1 \mathrm{mag}$ (dashed line). The UVOIR luminosities are shown with a dotted line. The luminosity corresponding to JD 2,453,565.5 is plotted with an error bar between $4.6 \times 10^{41}$ and $5.4 \times 10^{41} \mathrm{ergs} \mathrm{s}^{-1}$ owing to the large uncertainties in the $r^{\prime} i^{\prime}$ magnitudes.

Four late-time observations obtained with the Swope $1 \mathrm{~m}$ telescope between JD 2,453,538 and 2,453,570 involved only the $B V$ filters. In those cases, integration of the flux in the optical

\footnotetext{
${ }^{16}$ As a further corroboration, we included the few NIR photometry points in the BB fits. This exercise showed an agreement within $10 \%$ between the bolometric flux based on $u^{\prime}$ through $i^{\prime}$ and that based on $u^{\prime}$ through $K_{s}$ (or $u^{\prime}$ through $H$ on JD 2,453,483.5). The BB fits showed a systematic increase in temperature of about 500 to $800 \mathrm{~K}$ when going from a range of $B$ through $i^{\prime}$ to $B$ through $K_{s}$.
} 


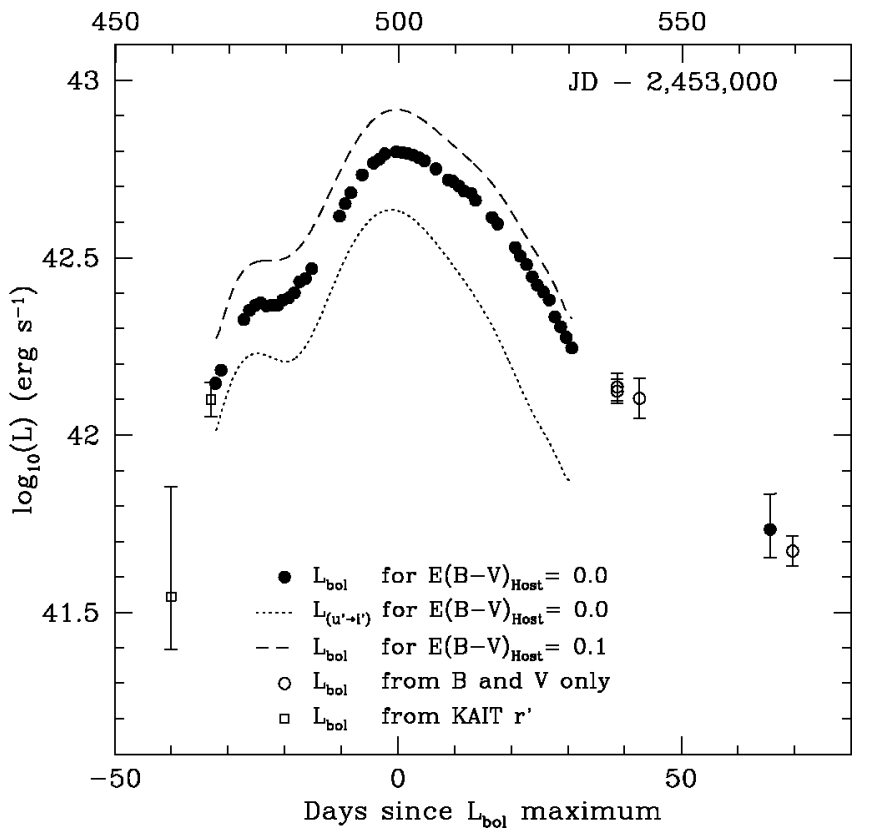

FIG. 8.-Bolometric light curve of SN $2005 \mathrm{bf}$ as derived from the optical photometry. Filled circles mark bolometric luminosities computed from integrated $u^{\prime} g^{\prime} r^{\prime} i^{\prime} B V$ fluxes, plus UV and IR corrections, $E(B-V)_{\text {Gal }}=0.045 \mathrm{mag}$, $E(B-V)_{\text {Host }}=0.0$, and an assumed distance of $83.8 \mathrm{Mpc}$. Open circles mark bolometric luminosities derived from observed $B-V$ colors and bolometric corrections. Open squares mark luminosities derived from $r^{\prime}$ magnitudes measured on the discovery images of KAIT and an extrapolation of the bolometric correction. The dashed line shows a smooth-curve fit to the bolometric luminosities obtained assuming $E(B-V)_{\text {Host }}=0.1 \mathrm{mag}$. The dotted line shows a smooth-curve fit to the UVOIR luminosities $L_{u^{\prime} \rightarrow i^{\prime}}$ derived in the range of $u^{\prime}$ through $i^{\prime}$, and assuming $E(B-V)_{\text {Host }}=0.0 \mathrm{mag}$.

range using the trapezoid approximation was not possible. Instead, bolometric luminosities were computed by determining bolometric corrections $\left(M_{\text {bol }}-V\right)$ from $B-V$ colors. A linear relation with a scatter of $\sim 0.03 \mathrm{mag}$ was found between these two quantities in the range $0.5<(B-V)<1.1 \mathrm{mag}$. The $B-V$ colors for the late-time epochs lie in the range from 1.04 to $1.18 \mathrm{mag}$. The relation above was used to derive the $L_{\text {bol }}$ values listed in parentheses in Table 2 . Figure 8 shows these values with open circles and error bars derived by propagating the uncertainties in $B-V$.

The observations obtained 30-70 days after maximum bolometric luminosity indicate late-time decline rates of $0.032 \pm$ $0.009 \mathrm{mag} \mathrm{day}^{-1}$ in $B, 0.034 \pm 0.004 \mathrm{mag} \mathrm{day}^{-1}$ in $V$, and $0.038 \pm 0.002 \mathrm{mag} \mathrm{day}^{-1}$ in $M_{\text {bol }}$. These values are significantly larger than the decline rate of $0.0098 \mathrm{mag} \mathrm{day}^{-1}$ predicted if all the energy from the decay of ${ }^{56} \mathrm{Co}$ into ${ }^{56} \mathrm{Fe}$ was fully thermalized in the ejecta (Woosley 1988), implying that at this epoch the ejecta of SN 2005 bf did not trap all the $\gamma$-ray radiation.

The discovery LOSS images from March 30 (JD 2,453,459.8) and April 6 (JD 2,453,466.8) can be used to provide valuable information on the early rise of the bolometric light curve. We consider here the case of $E(B-V)_{\text {Host }}=0.0 \mathrm{mag}$. The $r^{\prime}$ magnitudes derived from the unfiltered images were converted to bolometric magnitudes $\left(M_{\mathrm{bol}}\right)$ by extrapolating an average of the bolometric correction $\left(M_{\mathrm{bol}}-r^{\prime}\right)$ found for the first 10 days of follow-up observations with the Swope telescope (JD 2,453,467.7$2,453,477.6)$ to the discovery epochs. The resulting bolometric luminosities are given in parentheses in Table 2 and are plotted with open squares in Figure 8 . An uncertainty of $1.4 \times 10^{41} \mathrm{ergs}$ $\mathrm{s}^{-1}$ in the bolometric luminosity of April 6 was estimated by summing in quadrature the photometric error in $r^{\prime}$ and an un- certainty of $0.1 \mathrm{mag}$ in the adopted bolometric correction. In the case of March 30 the main source of error was the extrapolation of the bolometric correction. The uncertainty was estimated in this case by computing bolometric luminosities for BB curves of varying temperatures in the range $3000<T_{\mathrm{bb}}<20,000 \mathrm{~K}$ and passing through the $r^{\prime}$ flux point. This yielded an asymmetric error bar of $\left(2.50<L_{\text {bol }}<7.15\right) \times 10^{41} \mathrm{ergs} \mathrm{s}^{-1}$.

A simple integration of the bolometric luminosity between 40 days before and 35 days after the time of maximum luminosity yields a total bolometric energy output of $2.1 \times 10^{49}$ ergs.

\section{MODELS AND IMPLICATIONS}

Although SN 2005bf eventually evolved to resemble a fairly typical Type Ib SN, both its photometric and spectroscopic evolution leading up to maximum light were unusual in many respects, as follows:

1. The initial maximum observed in the $u^{\prime} g^{\prime} B V$ light curves is, to our knowledge, without precedent in Type $\mathrm{Ib} / \mathrm{Ic}$ light curves. Although it superficially resembles the shock outbreaks observed for the Type Ib/Ic SN 1999ex (Stritzinger et al. 2002) and the Type IIb SN 1993J (Richmond et al. 1994), in both of those cases the initial rise occurred very quickly ( $<2$ days), whereas the first maximum in SN 2005 bf occurred at least 2 weeks following outburst. This much longer rise time argues strongly against it being due to shock outbreak.

2. The bolometric light curve did not reach maximum until at least 40 days after outburst. Such a long rise time seems to be unique, although few Type Ib/Ic SNe have been caught early enough for us to be completely sure of this. We are aware of only two other Type Ic events, the hypernovae SN 1997ef and SN 1998 bw, which exhibited $V$ light curves of comparable width, although with shorter rise times (Galama et al. 1998; Iwamoto et al. 2000)

3. SN 2005 bf was also unusually luminous for a Type $\mathrm{Ib} / \mathrm{Ic}$ $\mathrm{SN}$, radiating $2.1 \times 10^{49} \mathrm{ergs}$ in the wavelength range longward of $3000 \AA$ during the first $\sim 75$ days following outburst (assuming spherical symmetry). This is nearly identical to the energy that we derived integrating the bolometric light curve of the hypernova SN 1998bw (Patat et al. 2001).

4. The transformation from a Type Ic SN at early epochs to a Type Ib SN by maximum light is also unprecedented. However, we must point out that few Type Ib/Ic SNe have been observed spectroscopically at such early epochs. It should be noted that if SN 2005bf had not been discovered until maximum light, it would have been classified as a fairly typical Type Ib SN. This serves to emphasize the importance of observing SNe as soon as possible after outburst.

5. The initial maximum in the $u^{\prime} g^{\prime} B V$ light curves was accompanied by the presence in the spectrum of high-velocity absorption lines of $\mathrm{Fe}$ II, $\mathrm{Ca}$ II, and $\mathrm{H}$ I. This absorption had disappeared by the time of the principal maximum in the bolometric light curve, which argues for it being physically associated with the mechanism responsible for the initial maximum.

6. The photospheric velocity of $9000-10,000 \mathrm{~km} \mathrm{~s}^{-1}$ observed at the early epochs ( $\sim 30$ days before maximum) was unusually low compared with typical Type Ib SNe (Branch et al. 2002).

7. None of the spectra presented here shows the narrow or intermediate-width emission lines caused by an interaction between the ejecta and any preceding slow circumstellar wind, as observed in Type Iln events (Schlegel 1990; Filippenko 1997).

The unique properties of SN 2005bf summarized above indicate an unusual event, one having certain features in common with ordinary Type $\mathrm{Ib} / \mathrm{c}$ SNe (see $\S 4.1$ ), Type Ilb events such as 
SN 1993J (Filippenko et al. 1993) and highly energetic SNe such as those seen in conjunction with GRBs 990425 (Galama et al. 1998) and 030329 (Hjorth et al. 2003; Stanek et al. 2003; Matheson et al. 2003). Both the high luminosity at the main peak and the long rise time argue for a more massive helium core and larger mass of ${ }^{56} \mathrm{Ni}$ than in ordinary Type $\mathrm{Ib} / \mathrm{Ic} \mathrm{SNe}$. Assuming a light curve dominated at peak by radioactive decay and complete trapping, to produce a luminosity of $6 \times 10^{42} \mathrm{ergs} \mathrm{s}^{-1} 40$ days after the explosion requires about $0.6 M_{\odot}$ of ${ }^{56} \mathrm{Co}$ (made as ${ }^{56} \mathrm{Ni}$ in the explosion). Such a large mass of ${ }^{56} \mathrm{Ni}$ is not produced in spherically symmetric explosions unless the explosion energy is very high, much greater than the canonical $10^{51} \mathrm{ergs}$, and the density gradient near the iron core is shallow, as in massive stars ( $225 M_{\odot}$; Woosley \& Weaver 1995).

On the other hand, the comparative faintness of the light curve during its first few days and the fact that the effective temperature was increasing at earlier times, rather than cooling, argue for a compact progenitor, neither a red nor blue supergiant. A red supergiant of any sort would be too bright and too hot initially and would cool monotonically with time. A blue supergiant with a radioactive peak around 40 days would be too faint on day 10 and would also have a declining temperature at early times.

The high-velocity hydrogen (with no low-velocity counterpart) shows that the star had lost most, but not all of its hydrogen envelope. The surface layers are shock accelerated to the highest speeds and a low mass of hydrogen would not mix extensively with the rest of the star. It seems that the progenitor was a WolfRayet star of spectral class WN (Maeder \& Meynet 1994) with less than $0.1 M_{\odot}$ of hydrogen in its outer layers.

Within these confines, we explored a variety of models, but found no physically reasonable solutions under the assumption of spherical symmetry and a monotonically decreasing radial abundance of ${ }^{56} \mathrm{Ni}$. The most successful one-dimensional model was the $2.0 \times 10^{51} \mathrm{ergs}$ explosion of an $8.29 M_{\odot}$ WN star with a mildly inverted distribution of ${ }^{56} \mathrm{Ni}$ with mass as shown in Figure 9. This star had a total ${ }^{56} \mathrm{Ni}$ mass of $0.6 M_{\odot}$, of which about $0.04 M_{\odot}$ was artificially sited in the helium shell. Except for the unusual "mixing," this was essentially the same helium and heavy-element core one obtains by evolving a $25 M_{\odot}$ mainsequence star to its end point. The vast bulk of the hydrogenic envelope was presumably lost to a binary companion quite late in the evolution. We did not conduct a broad survey of progenitor masses because we thought the restriction of doing onedimensional models did not warrant such an approach. It is possible that an acceptable model might also have been found for a higher mass helium core and larger explosion energy.

Roughly the outer $0.05 M_{\odot}$ of the WN star was composed of hydrogen and helium with mass fractions of 0.34 and 0.66 , respectively. Terminal velocities ranged from $13,000 \mathrm{~km} \mathrm{~s}^{-1}$ at the base of this "envelope" to over $30,000 \mathrm{~km} \mathrm{~s}^{-1}$ in the outer $0.001 M_{\odot}$. The rms velocity in the hydrogen-rich material was $18,000 \mathrm{~km} \mathrm{~s}^{-1}$. All calculations of presupernova evolution and explosive hydrodynamics up to the point of shock outbreak were done with the KEPLER implicit hydrodynamics package (Weaver et al. 1978; Woosley et al. 2002). The hydrogen envelope was removed at the end of helium burning and the star was in hydrostatic and thermal equilibrium at the time it exploded.

The light curve and multiband photometry of this model were then calculated using the STELLA code of Blinnikov et al. (1998) and Blinnikov \& Sorokina (2000). Although no fine-tuning was attempted, Figure 10 shows that this model gave a qualitatively good fit to both the observed bolometric luminosity and individual colors up until the main peak. To achieve reasonable agreement beyond the main peak, however, much greater $\gamma$-ray leakage

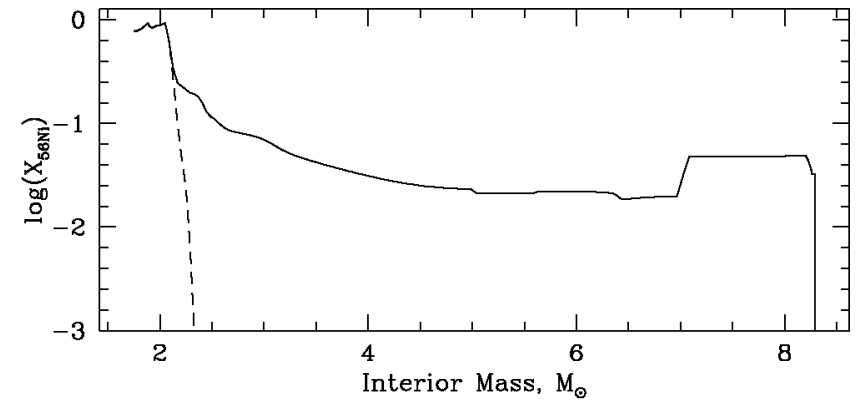

Fig. 9.-Distribution of the ${ }^{56} \mathrm{Ni}$ in the mixed (solid line) and unmixed (dashed line) versions of the $2 \times 10^{51} \mathrm{ergs}$ explosion of an $8.29 M_{\odot} \mathrm{WN}$ star. The mass fraction of ${ }^{56} \mathrm{Ni}$ is plotted as a function of the interior mass in the star.

had to be invoked than was calculated by STELLA for the spherically symmetric model. This was achieved by turning down the $\gamma$-ray opacity by a factor of 10 , while leaving the UVOIR opacities unaltered. During the rise to maximum this alteration had no effect, since the optical depth was very large. After the maximum, however, the decline rate was greatly accelerated. We were unable to find a model with unaltered $\gamma$-ray opacity in which the light curve peaked so late and yet declined so quickly.

All of these characteristics - the high explosion energy, large ${ }^{56} \mathrm{Ni}$ mass, inverted distribution of ${ }^{56} \mathrm{Ni}$, and the need for $\gamma$-ray leakage at late times-are suggestive of a grossly asymmetric explosion having many features in common with the supernovae found coincident with GRBs (e.g., Höflich et al. 1999; Mazzali et al. 2001, 2005; Maeda et al. 2003). In fact, SN 2005bf may be the best example so far of a "transition object" between the two classes of phenomena, namely, GRBs and ordinary Type Ib SNe.

A realistic simulation of such an event will only be achievable in a multidimensional simulation that captures the essence of energetic polar jets with milder mass ejection in the equatorial plane. Lacking at present the ability to do such a full multidimensional, relativistic simulation, including the necessary radiation transport, we also considered multicomponent models to reproduce the observed light curves. The scenario we considered was that of a WN progenitor star of about 6 to $15 M_{\odot}$ whose iron core collapses either to a black hole plus an accretion disk (Woosley 1993; MacFadyen \& Woosley 1999) or a very rapidly rotating neutron star (Wheeler et al. 2000). A relativistic jet was launched, as in current models for GRBs, but it was not observed, possibly because it was beamed to other angles or because it had too little energy in extremely relativistic ejecta. The jet was accompanied, however, by vigorous ${ }^{56} \mathrm{Ni}$-rich outflows extending out to approximately $45^{\circ}$ (MacFadyen \& Woosley 1999).

The polar regions thus experienced a much more violent explosion than the equator. A small fraction of the star's mass was ejected with very high velocity at both poles along the star's rotational axis. This material contained about $0.1 M_{\odot}$ of ${ }^{56} \mathrm{Ni}$ per pole, and the observer was situated somewhat off axis. The ensuing explosion can be separated into two components: (1) a polar explosion containing a small fraction of the total mass and moving at high velocity and (2) the explosion of the rest of the star. At first only the polar explosion is observed, producing the initial maximum; when that component fades and becomes transparent, the lower velocity ejecta become visible in the rise to the main maximum light.

As a specific example, by no means unique, we consider the same 8.29 $M_{\odot}$ WN star as before, but with no mixing of the ${ }^{56} \mathrm{Ni}$ beyond where it was produced in the one-dimensional model (Fig. 9, dashed line). In addition to this, we represent the higher velocity component by the $2 \times 10^{51} \mathrm{ergs}$ explosion of a $3.31 M_{\odot}$ 

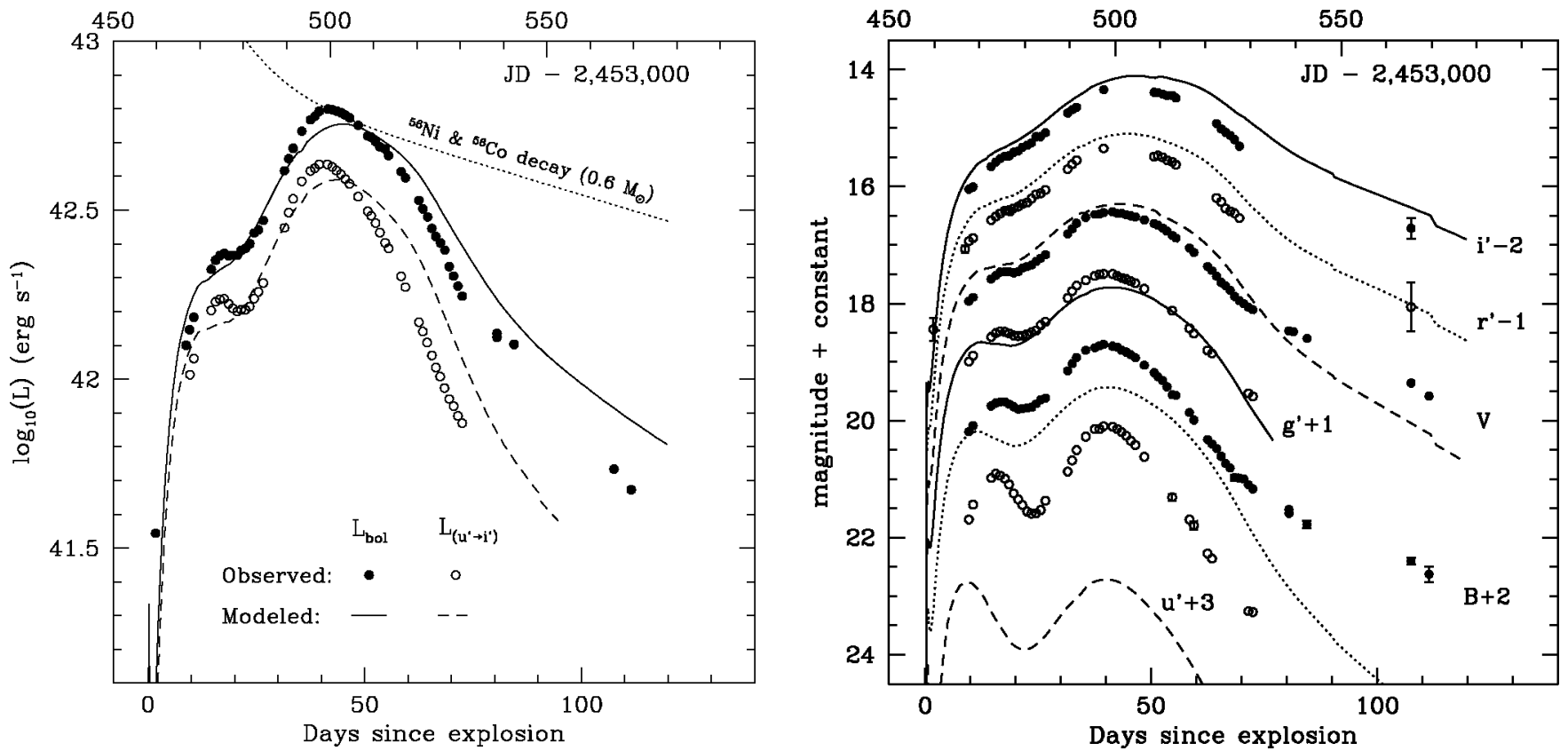

FIG. 10.-Left: Modeled bolometric (solid line) and UVOIR (dashed line) luminosities of the mixed $8.29 M_{\odot}$ WN star explosion. The circles mark the UVOIR

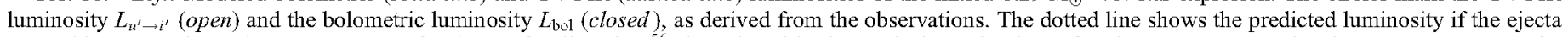

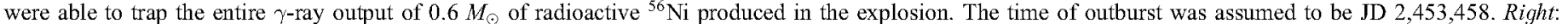
Modeled $u^{\prime} g^{\prime} r^{\prime} i^{\prime} B V$ light curves (lines) compared with the observations (circles)

WR star that left behind a $1.52 M_{\odot}$ remnant (hence the $2 \times$ $10^{51}$ ergs is concentrated in $1.79 M_{\odot}$ of ejecta). This component contains $0.1 M_{\odot}$ of ${ }^{56} \mathrm{Ni}$ (presumably at each pole). The composite light curve and colors are given in Figure 11. At early times ( $t<20$ days), before the rapidly expanding, low-mass component has become optically thin, the more slowly moving explosion is occulted and blocked from view. At late times ( $t>20$ days), the fast component is invisible. The geometry of the slower ejecta, viewed along the equator (side), resembles the number 8 . Gamma-rays can diffuse out much more effectively along the polar axis than along the equator. To account for this, the $\gamma$-ray opacity has been reduced by a factor of 10 .

\section{CONCLUSIONS}

The computer simulations presented here, although clearly suffering from being one-dimensional, strongly suggest that the light curve and the two-component spectrum of SN 2005bf can be qualitatively explained by the very energetic explosion of a
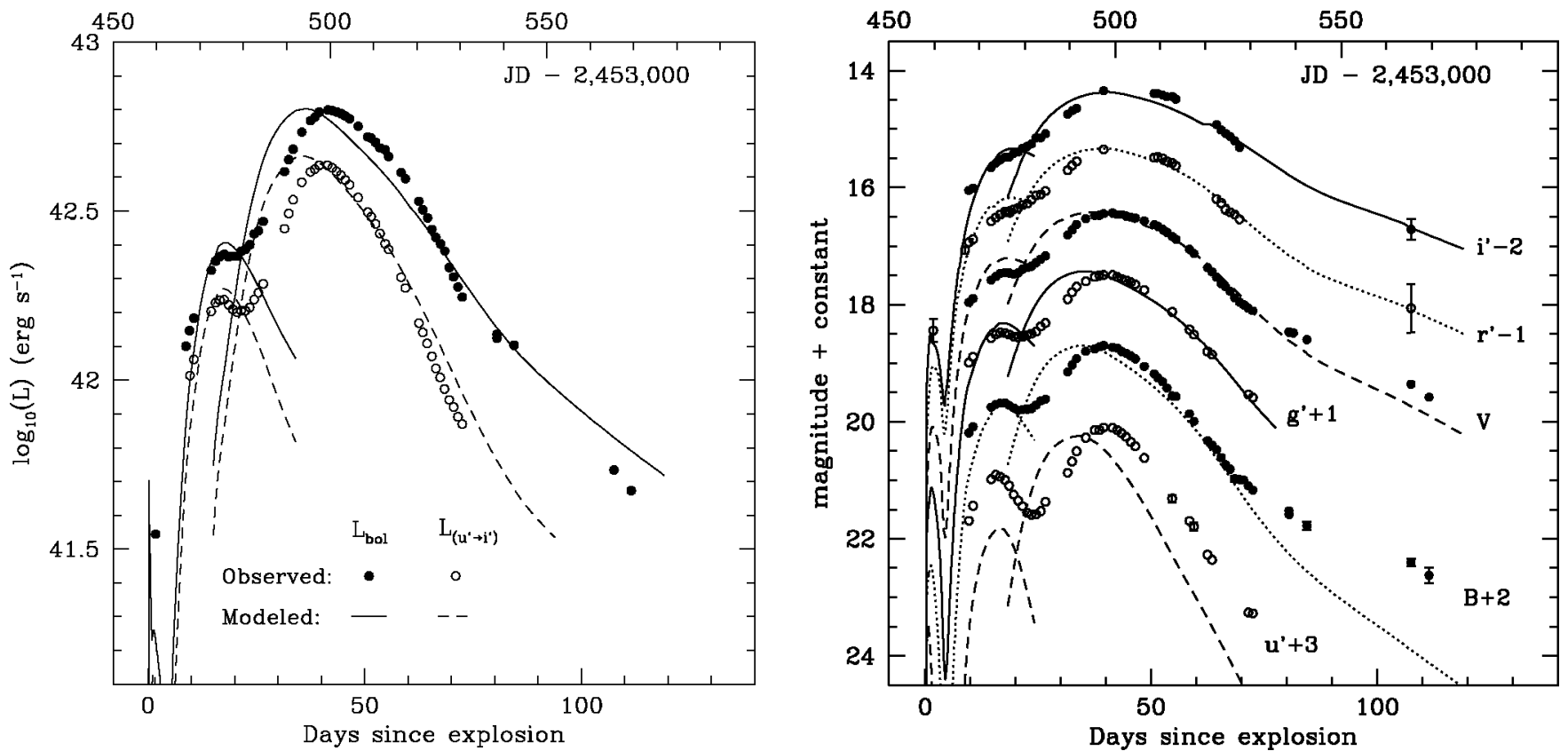

FIG. 11.-Left: Bolometric (solid line) and UVOIR (dashed line) luminosities of a composite model made of two explosions: $(a)$ the $2 \times 10^{51}$ ergs explosion of $1.72 M_{\odot}$ of helium and heavy elements containing $0.1 M_{\odot}$ of ${ }^{56} \mathrm{Ni}$, and $(b)$ the $2 \times 10^{51}$ ergs explosion of an $8.29 M_{\odot}$ WN star. Neither model had mixed ${ }^{56} \mathrm{Ni}$. The time of outburst was assumed to be ID 2,453,458. Component $a$ is observable until about 20 days after outburst, when it is overrun by component $b$. Right: Contributions of components $a$ and $b$ to the $u^{\prime} g^{\prime} r^{\prime} i^{\prime} B V$ light curves (lines) compared with the observations (circles). 
massive star that lost almost all of its hydrogen envelope and exploded very asymmetrically. The energy and ${ }^{56} \mathrm{Ni}$ mass implied are large compared with those of common Type $\mathrm{Ib} \mathrm{SNe}$, but the energy is intermediate between ordinary supernovae and so-called hypernovae, suggesting that SN $2005 \mathrm{bf}$ might be some sort of transition object between these two classes of phenomena. Given the high energy and the possible detection of polarization (Wang $\&$ Baade 2005), it is very likely that the explosion was asymmetric and will ultimately need to be modeled in at least two dimensions. The ${ }^{56} \mathrm{Ni}$ distribution could have been very aspherical, and this particularly complicates the modeling of the first peak.

Given the previously observed association of GRBs and energetic supernovae, it is quite plausible that SN 2005bf was powered by a central engine like those being discussed for GRBs. To retain the necessary degree of rotation, it is more likely that the envelope of the star was lost to a binary companion rather than in an ordinary standard wind. The longer the star remains a red supergiant, the more its core is braked by torques from the slowly rotating envelope (Heger et al. 2005; Woosley \& Heger 2006). Two-dimensional simulations should be conducted to verify the interpretation of this event as a possible "missing link." Radio observations to limit the amount of relativistic ejecta are also encouraged.

We would like to thank David Branch for allowing us to use SYNOW and Jerod Parrent for providing us with the code. We thank the referee for his/her valuable comments. This material is based on work supported by the National Science Foundation (NSF) under grant AST 03-06969. We also acknowledge support from Hubble Space Telescope grant GO-09860.07-A from the Space Telescope Science Institute, which is operated by the Association of Universities for Research in Astronomy, Inc., under NASA contract NAS5-26555. S. B. is supported in part by the RFBR grants 05-02-17480, 04-02-16793. B. L. L. was supported by an NSERC PGS B and a Walter C. Sumner Fellowship. M. H. acknowledges support provided by NASA through Hubble Fellowship grant HST-HF-01139.01-A. M. H. and G. G. are grateful for support from the Centro de Astrofísica FONDAP 15010003. A.V.F.'s group at the University of California, Berkeley, is supported by of NSF grant AST 03-07894; he is also grateful for a Miller Research Professorship, during which part of this work was completed. KAIT was made possible by generous donations from Sun Microsystems, Inc., the Hewlett-Packard Company, AutoScope Corporation, Lick Observatory, the NSF, the University of California, and the Sylvia and Jim Katzman Foundation. The W. M. Keck Observatory is operated as a scientific partnership among the California Institute of Technology (Caltech), the University of California, and NASA; the Observatory was made possible by the generous financial support of the W. M. Keck Foundation. This research has made use of the NASA/IPAC Extragalactic Database (NED), which is operated by the Jet Propulsion Laboratory, Caltech, under contract with NASA.
Allington-Smith, J., et al. 1994, PASP, 106, 983

Bessell, M. S. 1990, PASP, 102, 1181 1999, PASP, 111, 1426

Blinnikov, S. I., Eastman, R., Bartunov, O. S., Popolitov, V. A., \& Woosley, S. E. 1998, ApJ, 496, 454

Blinnikov, S. I., \& Sorokina, E. I. 2000, A\&A, 356, L30

Branch, D., et al. 2002, ApJ, 566, 1005

Cardelli, J. A., Clayton, G. C., \& Mathis, J .S. 1989, ApJ, 345, 245

Clocchiatti, A., \& Wheeler, J. C. 1997, ApJ, 491, 375

Falco, E. E., et al. 1999, PASP, 111, 438

Fisher, A. 2000, Ph.D. thesis, Univ. Oklahoma

Filippenko, A. V. 1982, PASP, 94, 715

1997, ARA\&A, 35, 309

2005, in The Fate of the Most Massive Stars, ed. R. Humphreys \& K. Stanek (San Francisco: ASP), 33

Filippenko, A. V., Li, W., Treffers, R. R., \& Modjaz, M. 2001, in SmallTelescope Astronomy on Global Scales, ed. W.-P. Chen, C. Lemme, \& B. Paczyński (San Francisco: ASP), 121

Filippenko, A. V., Matheson, T., \& Ho, L. C. 1993, ApJ, 415, L103

Filippenko, A. V., et al. 1995, ApJ, 450, L11

Foley, R. J., et al. 2003, PASP, 115, 1220

Freedman, W. L., et al. 2001, ApJ, 553, 47

Fukugita, M., Ichikawa, T., Gunn, J. E., Doi, M., Shimasaku, K., \& Schneider, D. P. 1996, AJ, 111, 1748

Galama, T. J., et al. 1998, Nature, 395, 670

Hamuy, M. 2004, in Stellar Collapse, ed. C. L. Fryer (Dordrecht: Kluwer), 39

Hamuy, M., Contreras, C., Gonzalez, S., \& Krzeminski, W. 2005, IAU Circ., 8520

Hamuy, M., Suntzeff, N. B., Heathcote, S. R., Walker, A. R., Gigoux, P., \& Phillips, M. M. 1994, PASP, 106, 566

Hamuy, M., et al. 2002, AJ, 124, 417 2006, PASP, 118, 2 (Paper I)

Harkness, R. P., et al. 1987, ApJ, 317, 355

Heger, A., Woosley, S. E., \& Spruit, H. C. 2005, ApJ, 626, 350

Hjorth, J., et al. 2003, Nature, 423, 847

Höflich, P., Wheeler, J. C., \& Wang, L. 1999, ApJ, 521, 179

Iwamoto, K., et al. 2000, ApJ, 534, 660

Landolt, A. U. 1992, AJ, 104, 340

Li, W., et al. 2000, in Cosmic Explosions, ed. S. S. Holt \& W. W. Zhang (New York: AIP), 103
REFERENCES

MacFadyen, A. I., \& Woosley, S. E. 1999, ApJ, 524, 262

Maeda, K., Mazzali, P. A., Deng, J., Nomoto, K., Yoshii, Y., Tomita, H., \& Kobayashi, Y. 2003, ApJ, 593, 931

Maeder, A., \& Meynet, G. 1994, A\&A, 287, 803

Martini, P., Persson, S. E., Murphy, D. C., Birk, C., Shectman, S. A., Gunnels,

S. M., \& Koch, E. 2004, Proc. SPIE, 5492, 1653

Matheson, T., et al. 2003, ApJ, 599, 394

Mazzali, P. A., Nomoto, K., Patat, F., \& Maeda, K. 2001, ApJ, 559, 1047

Mazzali, P. A., et al. 2005, Science, 308, 1284

Modjaz, M., Kirshner, R., \& Challis, P. 2005, IAU Circ., 8509

Monard, L. A. G., Moore, M., \& Li, W. 2005, IAU Circ., 8507

Morrell, N., Hamuy, M., Folatelli, G., \& Contreras, C. 2005, IAU Circ., 8509

Oke, J. B., et al. 1995, PASP, 107, 375

Panagia, N. 2003, in Lecture Notes in Physics, 598, Supernovae and Gamma

Ray Bursters, ed. K. W. Weiler (Berlin: Springer), 113

Patat, F., et al. 2001, ApJ, 555, 900

Persson, S. E., Murphy, D. C., Gunnels, S. M., Birk, C., Bagish, A., \& Koch, E. 2002, AJ, 124, 619

Persson, S. E., et al. 1998, AJ, 116, 2475

Richmond, M. W., et al. 1994, AJ, 107, 1022

Schlegel, D. J., Finkbeiner, D. P., \& Davis, M. 1998, ApJ, 500, 525

Schlegel, E. M. 1990, MNRAS, 244, 269

Smith, J. A., et al. 2002, AJ, 123, 2121

Stanek, K. Z., et al. 2003, ApJ, 591, L17 2005, ApJ, 626, L5

Stritzinger, M., et al. 2002, AJ, 124, 2100

Turatto, M., Benetti, S., \& Cappellaro, E. 2003, in From Twilight to Highlight: The Physics of Supernovae, ed. B. Leibundgut \& W. Hillebrandt (Berlin: Springer), 200

Wang, L., \& Baade, D. 2005, IAU Circ., 8521

Weaver, T. A., Zimmerman, G. B., \& Woosley, S. E. 1978, ApJ, 225, 1021

Wheeler, J. C., Harkness, R. P., Clocchiatti, A., Benetti, S., Brotherton, M. S.,

DePoy, E. L., \& Elias, J. 1994, ApJ, 436, L135

Wheeler, J. C., Yi, I., Höflich, P., \& Wang, L. 2000, ApJ, 537, 810

Woosley, S. E. 1988, ApJ, 330, 218 1993, ApJ, 405, 273

Woosley, S. E., \& Heger, A. 2006, ApJ, 637, 914

Woosley, S. E., Heger, A., \& Weaver, T. A. 2002, Rev. Mod. Phys., 74, 1015

Woosley, S. E., \& Weaver, T. A. 1995, ApJS, 101, 181 